\title{
Asymptotically Anti-de Sitter Spaces
}

\author{
Marc Henneaux $^{1, \star}$ and Claudio Teitelboim ${ }^{1,2}$ \\ 1 Center for Theoretical Physics, The University of Texas at Austin, Austin, TX 78712, USA \\ 2 Centro de Estudios Cientificos de Santiago, Casilla 16443, Santiago 9, Chile
}

\begin{abstract}
Asymptotically anti-de Sitter spaces are defined by boundary conditions on the gravitational field which obey the following criteria: (i) they are $\mathrm{O}(3,2)$ invariant; (ii) they make the $\mathrm{O}(3,2)$ surface integral charges finite; (iii) they include the Kerr-anti-de Sitter metric. An explicit expression of the $\mathrm{O}(3,2)$ charges in terms of the canonical variables is given. These charges are shown to close in the Dirac brackets according to the anti-de Sitter algebra. The results are extended to the case of $N=1$ supergravity. The coupling to gravity of a third-rank, completely antisymmetric, abelian gauge field is also considered. That coupling makes it possible to vary the cosmological constant and to compare the various anti-de Sitter spaces which are shown to have the same energy.
\end{abstract}

\section{Introduction}

The need for a satisfactory explanation of the vanishing of the cosmological constant is one of the outstanding problems of theoretical physics.

One feels that a possible way of making progress toward an eventual solution of the problem is to become familiar with one's enemy. Thus we investigate in detail in this paper the dynamics of the gravitational field when the cosmological constant is negative.

The reason for restricting ourselves to $A<0$ is that in that case spacelike surfaces are open (non-compact) and the system possesses a richer structure due to the presence of an asymptotic $\mathrm{O}(3,2)$ symmetry at spacelike infinity. It is then necessary to incorporate this asymptotic symmetry into the hamiltonian of the theory just as one incorporates the asymptotic Poincare group in the case $\Lambda=0$ [1]. This problem does not arise if $\Lambda>0$, since in that case the spatial sections are generically compact and there is no asymptotic structure.

* On leave from Département de Physique, Université Libre de Bruxelles, Belgium Chercheur qualifié du Fonds National Belge de la Recherche Scientifique 
"To incorporate the symmetry into the hamiltonian" means that one must find appropriate boundary conditions at infinity.

The first step in that direction is to observe that when $\Lambda<0$ the solution of the matter free Einstein equations possessing the maximum number of symmetries is the anti-de Sitter spacetime [2] whose group of motions is $\mathrm{O}(3,2)$. This geometry takes then the role played by Minkowski space when $\Lambda=0$. Therefore, one demands that the allowed fields in the action principle should approach at large spacelike distances the anti-de Sitter configuration.

However, this observation in itself is not sufficient. One must specify precisely what is meant by "approaching asymptotically the anti-de Sitter geometry." This must be done by finding a set of boundary conditions at spacelike infinity which satisfy the following requirements. (i) The asymptotic conditions must be invariant under the action of the anti-de Sitter group since otherwise a symmetry transformation would map an allowed configuration onto a non-allowed one. (ii) The symmetry transformations must have well defined canonical generators, i.e., one must be able to improve the naive weakly vanishing hamiltonian by adding to it appropriate surface integrals which make its functional derivatives well defined [1]. These generators should obey the $\mathrm{O}(3,2)$ algebra. (iii) The boundary conditions should include the asymptotically anti-de Sitter solutions of physical interest, such as the Kerr-anti-de Sitter metric [3], since otherwise, they would be too restrictive. (iv) Lastly, the boundary conditions should preferably be expressed directly in terms of the spacetime metric components, which are the variables to be summed over in the path integral.

Now, it is not satisfactory to simply mimic the boundary conditions of the $\Lambda=0$ case, that is, to require the allowed metrics to differ from the anti-de Sitter configuration, written in the standard coordinate system, by perturbation terms vanishing as $r^{-1}$ at infinity. Indeed, by doing so, one gets boundary conditions which not only do not obey requirement (i) above, but which also fail to make the $\mathrm{O}(3,2)$ surface integrals convergent.

Therefore, it is necessary to reconsider asymptotically anti-de Sitter spaces from the very beginning. This program is carried through in this paper.

We propose in the next section a precise definition of asymptotically anti-de Sitter spaces by means of boundary conditions at spatial infinity on the metric coefficients. These boundary conditions are explicitly shown to be $\mathrm{O}(3,2)$ invariant and to contain the Kerr-anti-de Sitter metric. They are also shown to imply the asymptotic conditions listed in [4], which were expressed in terms of the Ricci tensor components.

The canonical analysis is undertaken in Sects. III and IV, where the last necessary condition (ii) on the boundary conditions is proved to hold. An explicit surface integral formula for the $\mathrm{O}(3,2)$ charges is given in terms of the canonical variables of gravity. These surface integrals are all finite and close in the Dirac bracket according to the $\mathrm{O}(3,2)$ algebra.

New developments have been made recently in the study of the stability of the anti-de Sitter background [5-7]. Based on arguments similar to those of the $\Lambda=0$ case $[8,9]$, the anti-de Sitter solution has been shown, for a given $\Lambda$, to be a state of minimum energy. This result follows from the existence of an additional symmetry, namely, supersymmetry, under which the anti-de Sitter configuration is invariant 
(at least in the simpler models). Supersymmetry enables one to find a manifestly positive expression for the energy, by considering the variation of the supercharge under an infinitesimal global supersymmetry transformation [10-13].

These arguments remain formal in the $\Lambda<0$ case, however, as long as precise boundary conditions on the fermionic partners of the gravitational field are not given. These boundary conditions should obey criteria analogous to those of pure gravity, that is, they should be invariant under supersymmetry transformations [and under $\mathrm{O}(3,2)]$ and they should make the supercharge surface integral finite. It is only if these requirements are met that the graded extension of $\mathrm{O}(3,2)$ will be realized as a canonical (asymptotic) symmetry.

Again, one cannot fulfill the above demands if one simply takes over the boundary conditions of the $\Lambda=0$ case $\left[\psi_{\lambda}=O\left(r^{-2}\right)\right]$ and a detailed analysis is necessary.

That analysis is provided here for $N=1$ supergravity, along the same lines as for pure gravity. Boundary conditions under which the asymptotic $\operatorname{OSp}(1,4)$ symmetry is incorporated into the hamiltonian of the theory are given. The canonical generators of the $\operatorname{OSp}(1,4)$ superalgebra are shown to be well defined and, as an immediate consequence, they close in the Dirac bracket according to the $\mathrm{OSp}(1,4)$ superalgebra, so important in the proof of the positivity theorems. The extension to more elaborate models is not considered here but should proceed along the same conceptual lines.

Lastly, since the ultimate goal of the study of gravity with a cosmological constant is to understand why the latter vanishes, it is necessary to set up a formalism in which all anti-de Sitter configurations with different radii of curvature live in the same space, so that they can be compared.

We study that question in the last section, in the case when the mechanism that induces the cosmological constant is the coupling to gravity of an antisymmetric, third-rank, abelian gauge field [14]. The hamiltonian formalism is shown to be well defined even when one considers different values of $\Lambda$ simultaneously and the appropriate surface integrals are given. We find that when the theory consists only of gravity coupled to the antisymmetric tensor, all anti-de Sitter spaces possess classically the same $\mathrm{O}(3,2)$ charges (including the energy).

Finally, some useful information about the geometric properties of anti-de Sitter spaces and the Kerr-anti-de Sitter solution is collected in the appendices.

The results of this paper have been previously reported in abbreviated form in $[15,16]$.

\section{Asymptotically Anti-de Sitter Spacetimes}

As we mentioned in the introduction, the boundary conditions at spatial infinity should be devised so as to meet the following three requirements:

(i) they should contain the Kerr-anti-de Sitter metric [3], a metric which it is reasonable to take as the prototype which all isolated systems in anti-de Sitter space approach at infinity;

(ii) they should be invariant under the anti-de Sitter group $\mathrm{O}(3,2)$;

(iii) they should make the surface integrals associated with the generators of $\mathrm{O}(3,2)$ finite. 
For definiteness, we consider the coordinate systems in which the empty antide Sitter metric reads

$$
d s_{0}^{2}=-\left[1+\left(\frac{r}{R}\right)^{2}\right] d t^{2}+\left[1+\left(\frac{r}{R}\right)^{2}\right]^{-1} d r^{2}+r^{2} d \Omega^{2} .
$$

Here, $R$ is the radius of curvature, related to the cosmological constant $\Lambda$ by $R=(3 /-\Lambda)^{1 / 2}$. The components of the Killing vectors in that coordinate system as well as some of their properties are given in Appendix A.

One way to fulfill the conditions (i) and (ii) above is to start with the Kerr-antide Sitter metric, whose asymptotic structure is discussed in Appendix B, and to act on it with the $\mathrm{O}(3,2)$ group in all possible ways.

If one goes through that procedure, one generates metric perturbations $h_{\lambda \mu}$ $\left(d s^{2}=d s_{0}^{2}+h_{\lambda \mu} d x^{\lambda} d x^{\mu}\right)$ which behave asymptotically as

$$
\begin{aligned}
h_{t t} & =r^{-1} f_{t t}(t, \theta, \phi)+O\left(r^{-2}\right), \\
h_{t \theta} & =r^{-1} f_{t \theta}(t, \theta, \phi)+O\left(r^{-2}\right), \\
h_{t \phi} & =r^{-1} f_{t \phi}(t, \theta, \phi)+O\left(r^{-2}\right), \\
h_{t r} & =r^{-4} f_{t r}(t, \theta, \phi)+O\left(r^{-5}\right), \\
h_{r r} & =r^{-5} f_{r r}(t, \theta, \phi)+O\left(r^{-6}\right), \\
h_{r \theta} & =r^{-4} f_{r \theta}(t, \theta, \phi)+O\left(r^{-5}\right), \\
h_{r \phi} & =r^{-4} f_{r \phi}(t, \theta, \phi)+O\left(r^{-5}\right), \\
h_{\theta \theta} & =r^{-1} f_{\theta \theta}(t, \theta, \phi)+O\left(r^{-2}\right), \\
h_{\theta \phi} & =r^{-1} f_{\theta \phi}(t, \theta, \phi)+O\left(r^{-2}\right), \\
h_{\phi \phi} & =r^{-1} f_{\phi \phi}(t, \theta, \phi)+O\left(r^{-2}\right) .
\end{aligned}
$$

This motivates our adopting (II.2) as boundary conditions. One easily checks that these are $\mathrm{O}(3,2)$ invariant: the Lie derivative of the perturbation $h_{\lambda \mu}$ along the anti-de Sitter generators obeys the same boundary conditions as the perturbation itself. This can be easily seen by straightforward computations using the asymptotic behavior of the Killing vectors given in Appendix A [formulas (A.8a-c)]. [It is assumed throughout that $\partial_{r} O\left(r^{n}\right)=O\left(r^{n-1}\right)$ when needed.]

The above boundary conditions are actually invariant under Lie transport along a wider, infinite dimensional group generated by the vector fields $\xi^{\mu}$ obeying

$$
\xi^{\mu}-\frac{1}{2} \xi_{\infty}^{a b} U_{a b}^{\mu} \underset{r \rightarrow \infty}{\longrightarrow} 0 .
$$

Here, the components $\xi_{\infty}^{a b}$ of $\xi^{\mu}$ along the Killing fields $U_{a b}^{\mu}(a, b=1, \ldots, 5)$ are ten constants. The precise fall off of (II.3) is determined in Appendix C.

It will be shown in the next section that the transformations with $\xi_{\infty}^{a b}=0$, which vanish at infinity, have no surface integral associated with them and are accordingly pure ("proper" [17]) gauge transformations. Two invariance transformations differing by a pure gauge transformation, i.e., characterized by the same coefficients $\xi_{\infty}^{a b}$, must be identified since they produce the same physical effects. The 
quotient group so defined is just the anti-de Sitter group, which is in that sense the asymptotic symmetry group of the theory.

What happens here is quite different from the $\Lambda=0$ situation, where the asymptotic symmetry group contains not only the Poincare transformations but also angle-dependent translations ("supertranslations") when one adopts the usual $1 / r$ fall off of the metric to its Minkowskian value. The resulting group is called the Spi group and is analogous to the B.M.S. group [18].

A similar difference between $\Lambda=0$ and $\Lambda \neq 0$ occurs in the study of the asymptotic properties at null infinity, which coincides with spatial infinity in the $\Lambda<0$ case [2] and possesses ten conformal Killing vectors. These vectors correspond to the ten asymptotic isometries discussed above. When $\Lambda=0$, null infinity is a null surface and its degenerate induced metric possesses an infinity of conformal motions [19].

When $\Lambda=0$, one must impose stronger boundary conditions at infinity in order to get rid of the supertranslations. These can be parity conditions on the metric and its momentum [1] or asymptotic conditions on the magnetic part of the Weyl tensor [18]. (In the former case, not all supertranslations are eliminated, but because the remaining ones have a vanishing charge, they are effectively proper gauge transformations and there is no ambiguity in the Poincaré charges.)

But, when the cosmological constant is negative, there is no need to reinforce the natural boundary conditions (II.2) displayed above, since the anti-de Sitter group is selected by them as the asymptotic symmetry group. These boundary conditions are accordingly satisfactory as they stand, and will define what we mean by an asymptotically anti-de Sitter space. As discussed in the next section, they make all surface integrals associated with $\mathrm{O}(3,2)$ finite.

[It should be pointed out here that one can in fact relax (II.2d,f,g) and assume that the radial-angular components of the perturbation tend to zero at infinity as $r^{-2}$. This would not be a significant generalization, however, for one can always make these components behave as $r^{-4}$ at infinity by an appropriate "proper" spacetime coordinate transformation. Hence, these seemingly wider boundary conditions will not be considered here.]

The question immediately arises here as to the relation between (II.2) and the boundary conditions of $[20,6,4]$. In order to answer that question, we first note that the "physical metric"

$$
d s^{2}=d s_{0}^{2}+h_{\mu \nu} d x^{\mu} d x^{v}
$$

is conformally related to the metric $d \bar{s}^{2}$,

$$
d \bar{s}^{2}=\Omega^{2} d s^{2}, \quad \Omega=\left(\frac{r}{R}+1\right)^{-1}
$$

for which the surface at infinity $r=\infty$ - hereafter denoted by $I-$ is at a finite distance. Introducing the new coordinate

$$
x^{1^{\prime}}=1-\left(\frac{r}{R}+1\right)^{-1}
$$

(which is such that the coordinates of $I$-now defined by $x^{1^{\prime}}=1$ - are all finite), one then easily checks that: (i) $d \bar{s}^{2}$ is regular close to and on the surface at infinity; 
(ii) the induced metric on the surface at infinity is

$$
-d t^{2}+R^{2}\left(d \theta^{2}+\sin ^{2} \theta d \phi^{2}\right)
$$

and possesses accordingly $\mathrm{O}(3,2)$ as conformal group of motions; (iii) the gradient of $\Omega$ is space-like and of constant magnitude on $I$.

To complete the comparison with $[20,6,4]$, it remains to study the behavior of the Riemann tensor close to $I$. This is most easily carried out in the non-holonomic frame

$$
\begin{array}{rlrl}
\omega^{0} & =\left[1+\left(\frac{r}{R}\right)^{2}\right]^{1 / 2} d t, & \omega^{1} & =\left[1+\left(\frac{r}{R}\right)^{2}\right]^{-1 / 2} d r, \\
\omega^{2} & =r d \theta, & \omega^{3}=r \sin \theta d \phi .
\end{array}
$$

In that basis, the background metric has just the standard Minkowskian value whereas the perturbation components are of order $r^{-3}$, except $h_{10}, h_{12}$, and $h_{13}$, which tend to zero as $r^{-4}$.

Straightforward computations reveal that the Riemann tensor components of the physical metric with just one index equal to 1 (e.g., $R^{0}{ }_{123} \ldots$ ) generically go to zero at infinity as $r^{-4}$, while all the others (e.g., $R^{1}{ }_{010}$ or $R^{0}{ }_{203} \ldots$ ) typically differ from their asymptotic (background) value by terms of order $r^{-3}$.

This implies not only that the Ricci tensor components approach $\Lambda g_{\alpha \beta}$ as $r^{-3}$ but also that their covariant gradient goes to zero at the same rate. Hence, our boundary conditions obey the criteria for an asymptotically anti-de Sitter space listed in [4] (if one assumes in addition that $I$ is the whole of infinity, that is, there is only one asymptotic region). Conversely, it is shown in Appendix D that these criteria imply in turn that the metric obeys (II.2) in an appropriate coordinate system.

One can besides explicitly verify that the rescaled Weyl tensor $K_{\alpha \beta \gamma \delta}$ $\equiv \Omega^{-1} \bar{g}_{\alpha \lambda} C_{\beta \gamma \delta}^{\lambda}$ of the conformally related metric $d \bar{s}^{2}$ obey reflective boundary conditions of the type discussed in $[20,6]$ on the surface at infinity. This surface appears here as the space-time history of a "perfectly conducting" twodimensional surface, since the magnetic components of $K_{\alpha \beta \gamma \delta}$ with respect to $I$, (i.e., $K_{\alpha \beta \gamma \delta}$ with only one index equal to $\left.1^{\prime}\right)$ vanish on the surface at infinity, whereas its electric components $\left(K_{\alpha \beta \gamma \delta}\right.$ with two indices equal to $\left.1^{\prime}\right)$ remain finite. (The "dual" boundary conditions, which would also guarantee that no "information" leaks through $I$, are thus excluded, as demonstrated in [4].) .

We close this section by reformulating our basic boundary conditions (II.2) in terms of the canonical variables of general relativity, namely, the spatial metric $g_{i j}(x)$ and its conjugate momentum $\pi^{i j}(x)$. We consider here only spacelike hypersurfaces that go asymptotically to the $t=$ const hypersurfaces of (II.1) or to the hypersurfaces that can be obtained from them by an asymptotic anti-de Sitter transformation. On these slices, the momentum $\pi^{i j}(x)$ behaves asymptotically as

$$
\begin{aligned}
& \pi^{r r}(x)=r^{-1} p^{r r}(\theta, \phi)+O\left(r^{-2}\right), \\
& \pi^{r \theta}(x)=r^{-2} p^{r \theta}(\theta, \phi)+O\left(r^{-3}\right), \\
& \pi^{r \phi}(x)=r^{-2} p^{r \phi}(\theta, \phi)+O\left(r^{-3}\right),
\end{aligned}
$$




$$
\begin{aligned}
& \pi^{\theta \theta}(x)=r^{-5} p^{\theta \theta}(\theta, \phi)+O\left(r^{-6}\right), \\
& \pi^{\theta \phi}(x)=r^{-5} p^{\theta \phi}(\theta, \phi)+O\left(r^{-6}\right), \\
& \pi^{\phi \phi}(x)=r^{-5} p^{\phi \phi}(\theta, \phi)+O\left(r^{-6}\right),
\end{aligned}
$$

as it follows straightforwardly from its expression in terms of the metric and its spacetime derivatives.

Equations (II.9), together with the boundary conditions (II.2e--j) on the spatial metric constitute our starting point for the Hamiltonian analysis of the $\mathrm{O}(3,2)$ charges. [See Appendix $\mathrm{E}$ for a discussion of the preservation of the boundary conditions by the time evolution and for the need to strengthen somewhat (II.2e- $\mathrm{j}$ ) and (II.9).]

\section{Surface Integrals for the Anti-de Sitter Generators}

The hypersurface deformations defined by the asymptotic $\mathrm{O}(3,2) \mathrm{Killing}$ vector fields (II.3) are generated, in the canonical formalism, by the gravitational field hamiltonian $H[\xi]$

$$
H[\xi]=\int d^{3} x \xi^{\mu} \mathscr{H}_{\mu}+\frac{1}{2} \xi_{\infty}^{a b} J_{a b} .
$$

Here, the $\mathscr{H}_{\mu}(\mu=\perp, 1,2,3)$ are the usual constraint generators of general relativity [21-23],

$$
\begin{gathered}
\mathscr{H}_{\perp}=g^{-1 / 2}\left(\pi_{m n} \pi^{m n}-\frac{1}{2} \pi^{2}\right)-R g^{1 / 2}+2 \Lambda g^{1 / 2}, \\
\mathscr{H}_{i}=-2 \pi_{i ; j}^{j},
\end{gathered}
$$

the $\xi^{\mu}$ are the normal and tangential components of the deformation, and the $J_{a b}=-J_{b a}$ are surface integrals over a two-dimensional surface at infinity. In (III.2), the semicolon stands for the covariant differentiation in the spatial metric $g_{i j}$.

What determines the surface integrals $J_{a b}$ in the canonical formalism is the requirement that the hamiltonian (III.1) should have well defined functional derivatives when the canonical variables obey $($ II. 2,9$)$ and the deformation vector satisfies (II.3) [1]. In other words, its variation should be given only by a volume integral,

$$
\delta H[\xi]=\int d^{3} x\left[A^{i j}(x) \delta g_{i j}(x)+B_{i j}(x) \delta \pi^{i j}(x)\right],
$$

where the variations $\delta g_{i j}(x), \delta \pi^{i j}(x)$ of the canonical variables are compatible with the boundary conditions but are otherwise arbitrary. (The functions $A^{i j}(x)$ and $B_{i j}(x)$ are called the functional derivatives of $H[\xi]$ and can be found in [22].)

Now, if one computes the variation of the volume integral $\int d^{3} x \xi^{\mu} \mathscr{H}_{\mu}$ of (III.1), one finds, keeping all terms [1]

$$
\begin{aligned}
\delta \int d^{3} x \xi^{\mu} \mathscr{H}_{\mu}= & \int d^{3} x\left\{A^{i j}(x) \delta g_{i j}(x)+B_{i j}(x) \delta \pi^{i j}(x)\right\} \\
& -\oint d^{2} S_{l} G^{i j k l}\left(\xi^{\perp} \delta g_{i j ; k}-\xi^{\perp},{ }_{, k} \delta g_{i j}\right) \\
& -\oint d^{2} S_{l}\left\{2 \xi_{k} \delta \pi^{k l}+\left(2 \xi^{k} \pi^{j l}-\xi^{l} \pi^{j k}\right) \delta g_{j k}\right\},
\end{aligned}
$$


where

$$
G^{i j k l}=\frac{1}{2} g^{1 / 2}\left(g^{i k} g^{j l}+g^{i l} g^{j k}-2 g^{i j} g^{k l}\right) .
$$

The surface integral in (III.4) does not vanish for arbitrary $\delta g_{i j}, \delta \pi^{i j}$ obeying (II.2-9) - unless the $\xi_{\infty}^{a b}$ are all zero - and hence, cannot be neglected. However, it turns out that this surface term can be rewritten, under the assumed boundary conditions, as the variation of the following finite integral over a 2-sphere at infinity,

$$
-\frac{1}{2} \xi_{\infty}^{a b} J_{a b}
$$

with

$$
J_{a b}=\oint d^{2} S_{i}\left\{\dot{G}^{i j k l}\left[U_{a b}^{\perp} g_{k l \mid j}-U_{a b \mid j}^{\perp} h_{k l}\right]+2 U^{k}{ }_{a b} \pi_{k}^{i}\right\} .
$$

Here, the vertical bar denotes covariant differentiation in the spatial anti-de Sitter background $\stackrel{\circ}{g}_{i j}$ (II.1) and

$$
\stackrel{\circ}{G}^{i j k l}=\frac{1}{2} \stackrel{\circ}{ }^{1 / 2}\left(\stackrel{g}{g}^{i k} \stackrel{\circ}{ }^{j l}+\stackrel{\circ}{g}^{i l} \stackrel{o}{g}^{j k}-2 \dot{g}^{i j} \dot{g}^{k l}\right) .
$$

This suggests how to deal with the surface terms in (III.4). By adding to $\int d^{3} x \xi^{\mu} \mathscr{H}_{\mu}$ the surface integral $\frac{1}{2} \xi_{\infty}^{a b} J_{a b}$, as in (III.1), one gets a new "improved" hamiltonian which has well defined functional derivatives and which generates the correct equations of motion even for asymptotic $\mathrm{O}(3,2)$ surface deformations which do not become the identity at infinity $\left(\xi_{\infty}^{a b} \neq 0\right)$.

The improved hamiltonian does not vanish, even weakly. Its value on shell i.e., when the constraints hold - reduces to the surface term, which defines accordingly the $\mathrm{O}(3,2)$ charges. These charges are identical with those obtained in [5] by a different approach based on pseudo-tensors and superpotentials, if the latter are reexpressed in terms of canonical variables.

The use in (III.7) of the covariant derivative with respect to the background $g_{i j}$ explicitly shows that all $\mathrm{O}(3,2)$ charges $J_{a b}$ are invariant under spatial changes of coordinates and reduce to zero for the anti-de Sitter solution $\left(h_{k l}=\pi^{i j}=0\right)$. It also simplifies the technical analysis of the convergence of the integrals (III.7) as $r \rightarrow \infty$ : the surface terms with $g_{k l, j}$ and $g_{k m} \stackrel{\circ}{\Gamma}_{l j}^{m}$ separately diverge, but together, they remain finite.

It should be pointed out that the above method only determines the anti-de Sitter charges up to arbitrary constants $C_{a b}$ since only $\delta J_{a b}$ is involved in (III.4) and not $J_{a b}$ itself. These constants do not depend on $h_{i j}$ and $\pi^{i j}$, but could involve the radius of curvature $R$, which is kept constant in the present treatment. To evaluate $C_{a b}$ as a function of $R$, one must allow $R$ to vary. This will be done in the last section, where it will be shown that $C_{a b}$ is actually $R$ independent and hence can be chosen so that the charges $J_{a b}$ take the form (III.7) for all values of $R$. The choice (III.7) has the additional advantage of making the $J_{a b}$ obey the standard $\mathrm{O}(3,2)$ algebra without any (trivial) "central charge".

Lastly, we compute $J_{a b}$ both for the Schwarzschild-anti-de Sitter and the Kerranti-de Sitter solutions (Appendix B). In the first case, one has

$$
\begin{gathered}
h_{r r}=\frac{2 m R^{4}}{r^{5}}+O\left(r^{-8}\right), \text { other } h_{i j}=0, \\
\pi^{i j}=0,
\end{gathered}
$$


and formula (III.7) yields

$$
J_{51}=16 \pi m R \text {, other } J_{a b}=0 .
$$

In the second case, one finds (see Appendix B)

$$
\begin{gathered}
h_{\phi \phi}=\frac{2 m a^{2} \sin ^{4} \theta}{r}\left(1-\alpha^{2} \sin ^{2} \theta\right)^{-5 / 2}+O\left(r^{-3}\right), \\
h_{r r}=\frac{2 m R^{4}}{r^{5}}\left(1-\alpha^{2} \sin ^{2} \theta\right)^{-3 / 2}+O\left(r^{-7}\right), \\
\pi^{r \phi}=-\frac{3 a m \sin \theta}{r^{2}}\left(1-\alpha^{2} \sin ^{2} \theta\right)^{-5 / 2}+O\left(r^{-4}\right), \\
\alpha=\frac{a}{R}
\end{gathered}
$$

(the other components do not contribute to the surface integrals), and the nonvanishing charges are given by

$$
J_{51}=\frac{16 \pi m R}{\left(1-\alpha^{2}\right)^{2}}, \quad J_{23}=-\frac{16 \pi a m}{\left(1-\alpha^{2}\right)^{2}} .
$$

(The charges $J_{a b}$ all generate "rotations" and have consequently the dimensions of an angular momentum.) by

The $\mathrm{O}(3,2)$ Casimir invariants $I_{1}$ and $I_{2}$ (see Appendix B) turn out to be given

$$
\begin{aligned}
& I_{1}=\left[\frac{16 \pi m R}{\left(1-\alpha^{2}\right)^{2}}\right]^{2}\left(1+\alpha^{2}\right), \\
& I_{2}=-2\left[\frac{16 \pi m R}{\left(1-\alpha^{2}\right)^{2}}\right]^{4} \alpha^{2} .
\end{aligned}
$$

They both vanish when $m=0$; the Kerr solution reduces then to the exact anti-de Sitter background (see Appendix B). Upon contraction $R \rightarrow \infty$ ), the Kerr-anti-de Sitter metric becomes the usual Kerr metric. $J_{51} / R$ tends to the asymptotically flat space mass $16 \pi m$ whereas $J_{23}$ reduces to the ordinary angular momentum $-16 \pi a m$.

\section{Poisson Bracket Algebra}

The Poisson bracket algebra of the anti-de Sitter charges, which plays an important role in the proof of the positivity of the "energy" $J_{15}$ and of the stability of the anti-de Sitter solution, can be simply obtained here from geometric considerations.

Once the generator $H[\xi]$ is improved by the addition of the surface integral $\frac{1}{2} \xi_{\infty}^{a b} J_{a b}$, its functional derivatives with respect to the canonical variables become well defined. Moreover, the Poisson bracket of $H[\xi]$ and $H[\eta]$ [where $\xi^{\mu}$ and $\eta^{\mu}$ 
both define asymptotic $\mathrm{O}(2,3)$ surface deformations] becomes also well defined as a generator and obeys

$$
[H[\xi], H[\eta]]=H[[\xi, \eta]] .
$$

The bracket $[\xi, \eta]$ is given here by the "algebra of surface-deformations" [24],

$$
\begin{gathered}
{[\xi, \eta]=-\left(\eta^{i} \xi^{\perp},{ }_{i}-\xi^{i} \eta^{\perp}{ }_{, i}\right),} \\
{[\xi, \eta]^{i}=-g^{i j}\left(\eta^{\perp} \xi^{\perp}{ }_{, j}-\xi^{\perp} \eta^{\perp},{ }_{j}\right)+\left(\xi^{j} \eta^{i}{ }_{, j}-\eta^{j} \xi^{i},{ }_{j}\right) .}
\end{gathered}
$$

(That $[H[\xi], H[\eta]]$ has also well defined functional derivatives once $H[\xi]$ and $H[\eta]$ are improved can be checked by straightforward, but involved calculations.)

From (IV.2), one can express the asymptotic part of $[\xi, \eta]$ in terms of the asymptotic parts of $\xi$ and $\eta$. One then finds using (II.3) and (II.2) that $[\xi, \eta]$ also takes the form (II.3) and that, furthermore, the asymptotic part $[\xi, \eta]_{\infty}^{a b}$ is given in terms of $\xi_{\infty}^{a b}$ and $\eta_{\infty}^{a b}$ according to the Lie-algebra of $\mathrm{O}(3,2)$,

$$
[\xi, \eta]_{\infty}^{a b}=C^{a b}{ }_{c d e f} \xi_{\infty}^{c d} \eta_{\infty}^{e f} .
$$

This implies that the $\mathrm{O}(3,2)$ charges $H\left[U_{a b}^{\mu}\right]$ - which reduce to the surface term $J_{a b}$ when the constraints hold - indeed obey the $\mathrm{O}(3,2)$ algebra (in the Poisson bracket).

It is sometimes useful in applications to fix the gauge. In the case at hand this may be done by imposing conditions on $g_{i j}$ and $\pi^{i j}$ whose preservation under surface deformations destroys the possibility of an arbitrary $\xi^{\mu}$ inside. Thus, once the gauge is fixed, giving $\xi_{\infty}^{a b}$ determines $\xi^{\mu}$ everywhere.

The generators of the resulting overall "global" transformations are then just the surface integrals $J_{a b}$ by themselves. However, this time they must be understood as acting in terms of Dirac brackets $[25,23]$ associated with the chosen gauge condition. This is so because neither the volume integral nor the surface integral in (III.1) have separately well defined Poisson brackets but after the gauge is fixed the $\mathscr{H}_{\mu}$ become "strongly" (i.e., identically) zero and the $J_{a b}$ acquire well defined (Dirac) brackets.

As a consequence of the geometric arguments given above, one sees, without explicit computation of the change of the surface integral under a surface deformation obeying (II.3) that the Dirac brackets of the $J_{a b}$ close according to the algebra of $\mathrm{O}(3,2)$ and are thus independent of the particular gauge condition chosen.

\section{V. $N=1$ Supergravity}

The preceding analysis can be easily extended to supergravity. The framework and general conceptual points made above remain applicable but it becomes necessary to give also boundary conditions for the other fields appearing in the theory. Furthermore, $\mathrm{O}(3,2)$ is replaced by its appropriate graded extension as the asymptotic symmetry group. Additional surface integrals then appear which act as generators of the new symmetries. In those surface integrals, Killing spinors [5] play for the supersymmetries the role that Killing vectors play in (III.4). 
In the simplest case of $N=1$ supergravity with $\Lambda<0$ a vector spinor $\psi_{\lambda}$ is present as the fermionic partner of the metric $[26,27]$. The boundary conditions for $\psi_{\lambda}$ which accompany (II.2) turn out to be

$$
\begin{aligned}
\psi_{t} & =r^{-3 / 2}\left[1-\gamma_{(1)}\right] \chi_{t}(t, \theta, \phi)+O\left(r^{-5 / 2}\right), \\
\psi_{r} & =r^{-7 / 2}\left[1+\gamma_{(1)}\right] \chi_{r}(t, \theta, \phi)+O\left(r^{-9 / 2}\right), \\
\psi_{\theta} & =r^{-3 / 2}\left[1-\gamma_{(1)}\right] \chi_{\theta}(t, \theta, \phi)+O\left(r^{-5 / 2}\right), \\
\psi_{\phi} & =r^{-3 / 2}\left[1-\gamma_{(1)}\right] \chi_{\phi}(t, \theta, \phi)+O\left(r^{-5 / 2}\right),
\end{aligned}
$$

Here, the spinor indices are referred to the local orthonormal spherical frames defined below and $\gamma_{(1)} \equiv \gamma_{(r)}$ is the "radial" $\gamma$-matrix (whose square is unity). As a rule, indices in parentheses will refer to the orthonormal tetrads.

The actual local orthonormal spherical frames $e^{(\lambda)}{ }_{\mu}$ differ from their counterparts $\omega^{\lambda} \equiv e^{(\lambda)}$ (II.8) in the background metric by terms of the following order:

$$
\begin{aligned}
& e^{(r)}=e_{r}^{(r)}+O\left(r^{-4}\right), \\
& e^{(\hat{\lambda})}{ }_{\hat{\mu}}=e^{(\hat{\lambda})}{ }_{\hat{\mu}}+O\left(r^{-2}\right), \\
& e^{(\hat{\lambda})}{ }_{r}=e^{(\hat{\lambda})}{ }_{r}+O\left(r^{-5}\right), \\
& e^{(r)}{ }_{\hat{\lambda}}=e^{(r)}{ }_{\hat{\lambda}}+O\left(r^{-3}\right) .
\end{aligned}
$$

Here, the "hatted" indices refer to the angular variables $t, \theta, \phi$. These frames are only defined up to Lorentz rotations of order $r^{-3}$ in the angular-angular directions, and up to Lorentz rotations of order $r^{-4}$ in the radial-angular directions. For definiteness, we use in the calculations the following convenient frame:

$$
e_{\mu}^{(\lambda)}=e_{\mu}^{(\lambda)}+\frac{1}{2} e_{\alpha}^{(\lambda)} \stackrel{g}{g}^{\alpha \beta} h_{\mu \beta}+O\left(h^{2}\right) .
$$

(One easily checks that the ambiguity in the asymptotic Killing spinors resulting from the lack of complete determination of the frame $e^{(\lambda)}{ }_{\mu}$ is pure gauge - see below - and hence, has no physical effect.)

Straightforward computations show that the boundary conditions (V.1) are invariant under asymptotic anti-de Sitter transformations. Besides, one also sees that they are invariant under supersymmetry transformations

$$
\psi_{\lambda}^{\prime}-\psi_{\lambda}=\nabla_{\lambda} U-\frac{1}{2 R} \gamma_{\lambda} U+\text { torsion terms }
$$

where $U$ is a spinor field which becomes at infinity a linear combination of the Killing spinors $U_{A}$ (see Appendix A). The precise rate of approach is given by

$$
U=\xi_{\infty}^{A} U_{A}+\left(1+\gamma_{(1)}\right) a(t, \theta, \phi) r^{-5 / 2}+O\left(r^{-7 / 2}\right),
$$

where $a$ is an arbitrary $r$-independent spinor field. The following formula, valid asymptotically,

$$
\begin{aligned}
\text { right-hand-side of }(\mathrm{V} .4)= & h_{\mu \lambda \mid \varrho} S^{\mu \varrho} U-\frac{1}{4} h_{\lambda \mu} \gamma^{\mu} U \\
& + \text { torsion terms that die off faster }
\end{aligned}
$$

was found useful in explicitly verifying the invariance of (V.1) under (V.4). 
The boundary conditions (II.2d, f, g) on the metric components are, however, not invariant under the bosonic counterpart of (V.4),

$$
g_{\lambda \mu}^{\prime}=\bar{U} \gamma_{\lambda} \psi_{\mu}+\bar{U} \gamma_{\mu} \psi_{\lambda}
$$

for the radial-angular components $g_{r \hat{\lambda}}$ acquire through this transformation a $r^{-2}$ dependence.

But it turns out that this dependence can be compensated for by an appropriate coordinate transformation generated asymptotically by

$$
\begin{gathered}
\xi^{r}=0, \quad \xi^{\hat{\lambda}}=O\left(r^{-3}\right), \\
-\xi_{, r}^{\hat{\lambda}} \stackrel{\circ}{\hat{\lambda}} \hat{\mu} \hat{\mu}^{=} \bar{U} \gamma_{\hat{\mu}} \psi_{r}+\bar{U} \gamma_{r} \psi_{\hat{\mu}}+O\left(r^{-4}\right) .
\end{gathered}
$$

Such a coordinate transformation leaves invariant the other boundary conditions and does not change the surface integrals $J_{a b}$.

In other words "naive" supersymmetry transformations do not leave all the boundary conditions invariant since they fail to preserve the requirement $g_{r \hat{\lambda}}=O\left(r^{-4}\right)$. However, there are combined coordinate-supersymmetry transformations which are equivalent - i.e., which differ from (V.7) by a coordinate transformation without physical effects - and which preserve the asymptotic $r^{-4}$ fall off of $g_{r \hat{\lambda}}$. Equations (V.8) give just one of these transformations, which must replace the naive (asymptotically non-trivial) supersymmetry rotations when the boundary condition $g_{r \hat{\lambda}}=O\left(r^{-4}\right)$ is adopted, as here.

One can understand the need for the coordinate transformation (V.8) by realizing that the $r^{-2}$ and $r^{-3}$ parts of the angular-radial components $g_{r} \hat{\lambda}$ of the metric are pure gauge and can always be set equal to zero by an appropriate change of coordinates that tends to the identity at infinity. Once this is done, i.e., once the gauge condition $g_{r \hat{\lambda}}=O\left(r^{-4}\right)$ is enforced, one can only perform combined coordinate and supersymmetry transformations that preserve that gauge.

On account of the supersymmetry an extra term of the form

$$
\int d^{3} x \xi^{A} \mathscr{S}_{A}+\xi^{A}{ }_{\infty} Q_{A}
$$

is added to the hamiltonian (III.1), where the $\mathscr{S}_{A}$ are the constraint-generators of local supersymmetry transformations and $Q_{A}(A=1, \ldots, 4)$ are the supercharge surface integrals.

These surface integrals are again determined by demanding that (V.9) should have well defined functional derivatives. If one computes the variation of the volume integral $\int d^{3} x \xi^{A} \mathscr{S}_{A}$, one finds, keeping all terms,

$$
\begin{aligned}
\delta \int d^{3} x \xi^{A} \mathscr{S}_{A}= & \text { "volume terms" }-\oint d^{2} S_{i} \xi^{A}{ }_{\infty}\left\{g^{1 / 2} U_{A}^{T}\left[\gamma^{i}, \gamma^{j}\right] \delta \psi_{j}\right. \\
& +g^{1 / 2} U_{A}^{T}\left[\gamma^{i}, \gamma^{j}\right] S^{(a)(b)} \psi_{j} e_{(a)}{ }^{m} \delta e_{(b)} m \\
& \left.+g^{1 / 2} U_{A}^{T}\left[\gamma^{m}, \gamma^{j}\right] S^{(a)(b)} \psi_{j} e_{(a)}{ }^{k} e_{(b)}{ }^{i} \delta g_{k m}\right\}
\end{aligned}
$$

with $S^{(a)(b)}=\frac{1}{8}\left[\gamma^{(a)}, \gamma^{(b)}\right]$.

Under the above asymptotic decrease of the fields, the surface terms reduce to

$$
-\delta\left\{\xi_{\infty}^{A} \oint d^{2} S_{i} \dot{g}^{1 / 2} U_{A}^{T}\left[\dot{\gamma}^{i}, \dot{\gamma}^{j}\right] \psi_{j}\right\}
$$


Consequently, the supercharges $Q_{A}$ are given by

$$
Q_{A}=\oint d^{2} S_{i} \dot{g}^{1 / 2} U_{A}^{T}\left[\dot{\gamma}^{i}, \dot{\gamma}^{j}\right] \psi_{j},
$$

an expression formally equal to the $\Lambda=0$ one [8]. [ $\hat{\gamma}^{i}$ is equal to $\dot{e}_{(a)}{ }^{i} \gamma^{(a)}$ and $T$ denotes transposition. We have adopted the time gauge condition on the tetrads $\left(e_{(0) a}=0\right)$ for simplicity.]

The generators $J_{a b}, Q_{A}$ close in Dirac brackets according to the superalgebra of $\operatorname{OSp}(1,4)$

$$
\begin{aligned}
& {\left[J_{a b}, Q_{A}\right]=Q_{B}\left(N_{a b}\right)_{B A},} \\
& {\left[Q_{A}, Q_{B}\right]=\frac{i}{2} M_{A B}^{a b} J_{a b} .}
\end{aligned}
$$

Again here it is not necessary to resort to explicit computation to establish this algebra since it follows from the asymptotic form of the local supersymmetry algebra. (The matrices $M^{a b}, N_{a b}$ are given in Appendix A.)

\section{Varying the Cosmological Constant}

In the above analysis, the cosmological constant was treated as a fixed parameter and only those gravitational field configurations that approached asymptotically an anti-de Sitter space with a given radius of curvature were considered in the action principle.

It is much more satisfactory, however, to regard the cosmological constant as a constant of the motion rather than as a fundamental parameter appearing in the action of the gravitational field. This enables one to compare the various anti-de Sitter spaces which thus become different states of the same system. New possibilities are also opened up quantum mechanically.

In order to treat $\Lambda$ as a dynamical variable one must be able (by definition) to vary it in the action. This is not possible if one takes the usual gravitational action

$$
S=\int d^{4} x(-g)^{1 / 2}\left({ }^{(4)} R-2 \Lambda\right),
$$

because extremizing (VI.1) with respect to $\Lambda$ yields $g=0$, which is not permissible.

Therefore, a way is needed to include $\Lambda$ indirectly in the action. The simplest mechanism for doing this is suggested by supergravity [14]. It is given by coupling the gravitational field to a completely antisymmetric gauge field $A_{\mu v}$ according to

$$
S=\int d^{4} x(-g)^{1 / 2}\left({ }^{(4)} R+\frac{1}{2 \cdot 4 !} F_{\mu \nu \lambda Q} F^{\mu \nu \lambda \varrho}\right),
$$

where the gauge invariant field strength $F$ is given by the totally antisymmetric combination

$$
F_{\mu v \varrho \sigma}=\partial_{[\mu} A_{v \varrho \sigma]} .
$$

As will be shown shortly, the coupling to gravity of this four-dimensional generalization of the Schwinger model is equivalent to the inclusion of a negative 
cosmological constant. [The case $\Lambda>0$ can be described by changing the sign of the coupling in (VI.2), but it will not be discussed here since it does not raise boundary term difficulties at spatial infinity. Indeed, when $\Lambda>0$, it is natural to consider only closed spacelike sections.]

We now pass to discuss the relationship between the action principles based on (VI.1) and (VI.2) within the canonical formalism. The hamiltonian which follows from (VI.2) reads

$$
H=\int d^{3} x\left(\xi^{\mu} \mathscr{H}_{\mu}+\xi_{i j} \gamma^{i j}\right)+(\text { surface integral }) .
$$

Here $\mathscr{H}_{\mu}$ is the sum of the gravitational constraint-generators (with $\Lambda=0$ ) and of the energy momentum densities of the gauge field which are

$$
\begin{aligned}
& \mathscr{H}_{\perp}^{\text {gauge field }}=-\frac{1}{2 \cdot 3 !} g^{-1 / 2} \pi^{i j k} \pi_{i j k}, \\
& \mathscr{H}_{i}^{\text {gauge field }}=0 .
\end{aligned}
$$

In (VI.5), $\pi^{i j k}$ is the gauge field momentum conjugate to the spatial component $A_{i j k}$. The function $\gamma^{i j}$ is the constraint-generator of the gauge transformations

$$
A_{\mu \nu \varrho} \rightarrow A_{\mu v \varrho}+\partial_{[\mu} \Lambda_{v \varrho]},
$$

under which the action is invariant. It is given by

$$
\gamma^{i j}=-\pi_{, k}^{i j k} \text {. }
$$

The field component $A_{o i j}$ enters the action as a Lagrange multiplier for the constraint-generator (VI.6b). It is the $\xi_{i j}$ appearing in (VI.4).

When the equations for the gauge field are satisfied, the field strength $\pi^{i j k}$ takes the form

$$
\pi^{i j k}=\frac{2 \sqrt{3}}{R} \varepsilon^{i j k},
$$

where $R$ is independent of space and time and where $\varepsilon^{i j k}$ is the three-dimensional Levi-Civita tensor density. If one inserts (VI.7) back into the hamiltonian, one sees that a cosmological constant,

$$
\Lambda=-\frac{3}{R^{2}}
$$

appears as a constant of integration in the equations of motion of the coupled system of gravitational and gauge fields.

What happens here is that due to the gauge invariance of the action, the gauge field has only one degree of freedom (not one per space point, just one). By virtue of the field equations, the canonical coordinate that describes that single degree of freedom is then constant in time. From the point of view of the metric (i.e., in Einstein equations), different values of that constant of the motion play the role of different cosmological constants. In this sense the theory with the anti-symmetric gauge field and without cosmological constant is equivalent to the Einstein theory with an arbitrary cosmological constant and without anti-symmetric gauge field. 
However, the very fact that the cosmological constant appears as an integration constant that can take any (negative) value will enable one to compare the anti-de Sitter spaces with different radii of curvature, which correspond, in this theory, to different configurations of the same system. The comparison cannot be even attempted when $\Lambda$ is given a priori as a universal constant because then only one anti-de Sitter space is a solution, that one whose radius of curvature is related to the universal $\Lambda$ through $\Lambda R^{2}=-3$.

The key point in making the comparison actually possible is the existence of a surface integral in the Hamiltonian (VI.4) which makes its functional derivatives - and hence those of the action - well defined. This surface integral determines the $\mathrm{O}(3,2)$ charges.

The need to have well defined functional derivatives of the action is not a technicality but it is a fundamental point. It ensures that when the field equations hold, the action is indeed stationary under the most general variation of the fields consistent with the boundary conditions.

Because we do not want to impose a particular value to the cosmological constant through the boundary conditions at spatial infinity, we must enlarge the class of field configurations of the previous sections so as to include all anti-de Sitter spaces, and not just one with a given radius of curvature.

In order to carry out this program, we rewrite the metric $g_{\lambda \mu}$ in terms of dimensionless coordinates $\tau=t / R, \varrho=r / R, \theta, \phi$. Next, we set

$$
d s^{2}=R^{2}\left(\stackrel{\circ}{g}_{\lambda \mu}+h_{\lambda \mu}\right) d x^{\lambda} d x^{\mu},
$$

where $\stackrel{\circ}{\lambda \mu}_{\lambda \mu}$ and $h_{\lambda \mu}$ are also dimensionless and are obtained from $g_{\lambda \mu}$ and $h_{\lambda \mu}$ of the previous sections by taking $R=1$. The perturbation $h_{\lambda \mu}$ obeys the boundary conditions (II.2). The key new feature is that the radius of curvature $R$ appearing in (VI.9a) is no longer a universal constant but can take any value. That value may, in principle, be time dependent. In order to preserve the canonical commutation relations, we also redefine

$$
\pi^{i j}=R^{-2}\left(\pi^{i j} \text { of Sect. II with } R=1\right) .
$$

The boundary conditions on the gauge field momentum are taken to be

$$
\pi^{i j k}=\frac{2 \sqrt{3}}{R} \varepsilon^{i j k}+O\left(\varrho^{-4}\right)
$$

with the same (unspecified) $R$ as in (VI.9a). This ensures that the constraints hold asymptotically. The gauge field itself will be restricted to vanish as $\varrho^{-1}$ at infinity,

$$
A_{i j k}=\varepsilon_{i j k} \frac{a(t, \theta, \phi)}{\varrho}+O\left(\varrho^{-2}\right) .
$$

(The behavior of the Lagrange multiplier $A_{o i j}$ will be determined later on.)

Since the functional space defined here is larger than the one considered in the previous sections, it is necessary to re-examine the variation of $H$. The new class of field variations might indeed yield new surface terms to be added to the hamiltonian (or might, a priori, make the formalism inconsistent if no surface term at all could be found that would make $H$ to have well defined functional derivatives). 
These new variations are just overall conformal changes in the metric $g_{i j}$, induced by varying the radius of curvature in (VI.9a), and do not vanish at infinity. In order to analyze their role, we first study the variation of $H$ under a change of $R$ only, from one pure anti-de Sitter configuration $\left[\stackrel{\circ}{g}_{i j}=R^{2} \stackrel{\circ}{g}_{i j}, h_{i j}=0, \pi^{i j}=0\right.$, $\left.\pi^{i j k}=(2 \sqrt{3} / R) \varepsilon^{i j k}\right]$ to another. This simpler case contains the main points. Besides, it illustrates very clearly that all anti-de Sitter spaces have the same $\mathrm{O}(3,2)$ charges [the variation $H$ will turn out to be zero under $\mathrm{O}(3,2)$ surface deformations].

It is easy to see from (III.4) that the variation of $\int d^{3} x \xi^{\mu} \mathscr{H}_{\mu}-$ where $\xi^{\mu}$ obeys (II.3) - under $\delta g_{i j}=2 R \delta R \stackrel{g}{i j}_{i j}$, consists of the usual volume term plus a surface integral at infinity. When the variation $2 R \delta R \stackrel{g}{i j}_{i j}$ is made away from a pure anti-de Sitter configuration, this surface integral reduces to

$$
-2 R \delta R \xi_{\infty}^{a b} \oint d^{2} S_{k}(\stackrel{\circ}{g})^{1 / 2} U_{a b \mid m}^{\perp} \stackrel{\circ}{g}^{k m},
$$

and diverges like $\varrho^{3}$ when the Killing vector $U_{a b}^{\mu}$ generates time translations. (Here, $U_{a b}^{\perp}$ is equal to $U_{a b}^{\perp}$ of the previous sections rescaled by the factor $R^{-1}$ so that it is dimensionless. The parameters $\xi_{\infty}^{a b}$ are also dimensionless and $H$ has the dimensions of an angular momentum.)

The divergence of the surface integral (VI.11) does not mean that there is no hamiltonian formulation when the cosmological constant is varied. For, another surface integral arises from the variation of the gauge-generator $\gamma^{i j}$. This boundary contribution reads explicitly

$$
-\oint d^{2} S_{k} \xi_{i j} \delta \pi^{i j k}=\frac{2 \sqrt{3} \delta R}{R^{2}} \oint d^{2} S_{k} \varepsilon^{i j k} \xi_{i j}
$$

By taking $\xi_{i j}$ equal to

$$
\xi_{i j}=\frac{1}{2} \frac{R^{3}}{\sqrt{3}} \xi_{\infty}^{a b} U_{a b \mid m}^{\perp} \grave{g}^{k m}(\stackrel{g}{)})^{1 / 2} \varepsilon_{k i j},
$$

the integral (VI.12) cancels exactly (VI.11), so that the surface term in the variation of $\int d^{3} x\left(\xi^{\mu} \mathscr{H}_{\mu}+\xi_{i j} \gamma^{i j}\right)$ is actually equal to zero. Since this surface term must also be equal to minus the variation of the $\mathrm{O}(3,2)$ charges (times $\left.\xi_{\infty}^{a b}\right)$ between two anti-de Sitter configurations, one concludes furthermore that these configurations possess exactly the same anti-de Sitter charges.

The adjustment (VI.13), dictated by the desire to have a well-defined canonical formulation of the theory even when $\Lambda$ is varied, can also be arrived at by different considerations, as we now pass to show. These considerations will also reveal that the choice (VI.13) is unique if one wants the surface deformation algebra to become asymptotically that of $\mathrm{O}(3,2)$.

The point here is that the boundary condition (VI.10b) on the gauge field $A_{i j k}$ is not invariant under a naive $\mathrm{O}(3,2)$ surface deformation. Indeed, the variation of $A_{i j k}$ reads

$$
\delta A_{i j k}=-\frac{1}{3 !} R^{3} \sqrt{3} \xi_{\infty}^{a b} U_{a b}^{\perp} \stackrel{\circ}{ }^{1 / 2} \varepsilon_{i j k}
$$

an expression which grows as $\varrho^{2}$ at infinity (except for a spatial rotation for which it vanishes). Therefore, a naive anti-de Sitter transformation cannot be allowed in the present formalism, since it does not preserve the asymptotic behavior of the fields. 
The transformation (VI.14) can, however, be exactly compensated for by an appropriate gauge transformation, which turns out to be precisely given by (VI.13) because $\xi^{\perp}=(1 / 2) \xi_{\infty}^{a b} U_{a b}^{\perp}$ obeys

$$
\dot{g}^{i} i \stackrel{\circ}{V}_{i} \grave{P}_{j} \xi^{\perp}=3 \xi^{\perp}
$$

as a consequence of Killing's equation for the anti-de Sitter metric. Accordingly, although neither naive $\mathrm{O}(3,2)$ surface deformations nor gauge transformations of the type (VI.13) can be considered separately, they become admissible transformations when they are performed simultaneously.

Moreover, these combined transformations clearly obey the algebra of $\mathrm{O}(3,2)$ since they do not affect the gauge field variables at all [this property makes (VI.13) unique] and they coincide with ordinary deformations for the gravitational variables. Hence, by locking through (VI.13) a "compensating gauge transformation" with every surface deformation, one recovers $\mathrm{O}(3,2)$ as an asymptotic symmetry group.

The extension of these results to the general case (variation of $H$ away from an arbitrary configuration obeying the boundary conditions) follows the same line of thinking but raises some technical complications which we merely summarize.

One again finds that the $\mathrm{O}(3,2)$ surface deformations must be accompanied by a compensating gauge transformation $\xi_{i j}^{\text {comp }}[\xi]$ asymptotically given by

$$
\xi_{i j}^{\text {comp }}[\xi]=\xi^{(1)}{ }_{i j}+\xi^{(2)}{ }_{i j} \text {. }
$$

The first term in the right-hand-side of (VI.16a) is of order $\varrho^{3}$ and is the obvious generalization of (VI.13),

$$
\xi^{(1)}{ }_{i j}=\frac{1}{2} \frac{R^{2}}{\sqrt{3}} \xi_{\infty}^{a b} U_{a b \mid m}^{\perp} g^{k m}(g)^{1 / 2} \varepsilon_{k i j} .
$$

Just as in the pure anti-de Sitter case considered above, the gauge transformation associated with it compensates - but, this time, only to order $\frac{1}{\varrho}$ - the unwanted variation of $A_{i j k}$ induced by the term $-(1 / 2)(g)^{-1 / 2} \pi_{i j k} \pi^{i j k}$ in the hamiltonian generator $\mathscr{H}_{\perp}\left[\Delta \xi^{\perp}=3 R^{-2} \xi^{\perp}+O\left(\varrho^{-2}\right)\right]$.

The need for $\xi^{(2)}{ }_{i j}$, which yields finite surface integrals and only affects $A_{i j k}$ to order $1 / \varrho$, is more subtle. It results from the fact that the total surface term arising from the variation of the volume piece of the hamiltonian, which is finite once (VI.16b) is included, is not an "exact differential", i.e., the variation of a surface integral, if $\xi^{(2)}{ }_{i j}=0$. This is so because the coefficient of $\delta R$ has not then the appropriate (finite) functional dependence on the other independent variables $h_{i j}, \pi^{i j}$. But this coefficient can be adjusted at will to be a suitable choice of $\xi_{i j}$ in (VI.12). This fixes the asymptotic form of $\xi^{(2)}{ }_{i j}$ uniquely if one requires in addition that $\xi^{(2)}{ }_{i j}$ reduces to zero for the anti-de Sitter space. The parameter $\xi^{(2)}{ }_{i j}$ is asymptotically a function of $h_{i j}$ and $h_{i j, k}$ and its variation does not contribute to the surface terms because $\xi^{(2)}{ }_{i j}$ is multiplied by $\gamma^{i j}$, which vanishes as $\varrho^{-4}$. The explicit form is,

$$
\xi^{(2)}{ }_{i j}=-\frac{R^{3}}{4 \sqrt{3}} \varepsilon_{i j k} \xi_{\infty}^{a b} \dot{G}^{k l m n}\left(U_{a b}^{\perp} g_{m n \mid l}-U_{a b \mid l}^{\perp} h_{m n}\right)
$$


After $\xi_{i j}^{\text {comp }}[\xi]$ is included, the surface term in the variation of $\int d^{3} x\left(\xi^{\mu} \mathscr{H}_{\mu}+\xi_{i j} \gamma^{i j}\right)$ takes the form

$$
-\delta\left(\frac{1}{2} \xi_{\infty}^{a b} J_{a b}+\int \bar{\xi}_{i j} \pi^{i j k} d^{2} S_{k}\right)
$$

where the $J_{a b}$ are the same functions of the perturbation $h_{i j}$ from the anti-de Sitter geometry as above,

$$
J_{a b}=R^{2} \oint d^{2} S_{i}\left\{G^{i j k l} U_{a b}^{\perp} h_{k l \mid j}-U_{a b \mid j}^{\perp} h_{k l}+2 U_{a b}^{k} \pi_{k}^{i}\right\} .
$$

The parameter $\bar{\xi}_{i j}$ in (VI.17) is independent of $\xi^{\mu}$. It is equal to the difference between the $\xi_{i j}$ appearing in (VI.4) and the compensating gauge totation $\xi_{i j}^{\text {comp }}[\xi]$ needed to bring the surface integral to the form (VI.17). Thus, except for the restrictions implied on its asymptotic behavior by the preservation of (VI.10b) under gauge transformations, $\bar{\xi}_{i j}$ is an arbitrary function of $x$ and $t$. It contains the gauge freedom of the system.

There are two conceptually different kinds of gauge transformation contained in $\bar{\xi}_{i j}$. If the term

$$
\oint \bar{\xi}_{i j} \pi^{i j k} d^{2} S_{k}
$$

is different from zero, then one has a "global gauge rotation" and (VI.18) gives the "total associated charge"

$$
Q=\frac{R^{2}}{3}
$$

multiplied by the amount of asymptotic internal rotation

$$
\alpha_{\infty}=\frac{6 \sqrt{3}}{R^{3}} \oint \bar{\xi}_{i j} \varepsilon^{i j k} d^{2} S_{k} .
$$

The cosmological constant is just $-Q^{-1}$. (We have normalized the charge $Q$ so that it has also the dimensions of an angular momentum.)

A gauge transformation with a non-zero value for (VI.20) should be thought of a priori as inducing a change in the physical state. Such a transformation, which has been termed "improper" in [17], is quite analogous to a surface deformation (II.3) with a non-zero $\xi_{\infty}^{a b}$.

On the other hand, if for a given $\bar{\xi}_{i j}$ the flux (VI.20) is zero (as it happens when $\bar{\xi}_{i j}$ leaves the potential $A_{i j k}$ invariant everywhere in space), then the gauge transformation is a bona-fide "proper" one which can have no physical effect. It is only this latter proper gauge freedom which can legitimately be eliminated by eventually imposing a gauge condition.

It should be noted at this point that the boundary conditions adopted here ( $A_{i j k} \rightarrow 0$ at infinity) differ from more traditional gauge choices $\left(A_{o i j}=0, A_{i j k} \sim \varrho\right.$, see e.g. [28]) by an improper gauge transformation yielding an infinite asymptotic amount of rotation $\alpha_{\infty}$ (but the gauge invariant field strength is, of course, the same). Therefore, the latter gauges are not permissible in our formalism.

It follows from (VI.17) that the complete hamiltonian (VI.4) whose functional derivatives are well defined reads explicitly

$$
H=\int d^{3} x\left(\xi^{\mu} \mathscr{H}_{\mu}+\xi_{i j}^{\text {comp }}[\xi] \gamma^{i j}+\bar{\xi}_{i j} \gamma^{i j}\right)+\xi_{\infty}^{a b} J_{a b}+\alpha_{\infty} Q
$$


The charges $J_{a b}$ are exactly the same as when the cosmological constant is a universal constant, i.e., unvaried. They commute with the internal charge $Q$. By the same arguments as those used above, they can be shown to obey the $O(3,2)$ algebra, without the "central charge" $Q$ on the right-hand side. For this reason these $J_{a b}$ will be called the $\mathrm{O}(3,2)$ charges even when the cosmological constant is varied.

The need to include a "compensating gauge transformation" in the canonical formalism in order to have well defined generators for the asymptotic $\mathrm{O}(3,2)$ symmetry means that one must replace one's naive notion of a spacetime displacement by an appropriate combination of surface deformations and gauge transformations. This is not the first instance of such an interplay between displacements in "physical space" and "internal space". A similar phenomenon happens in the field of a magnetic monopole [29] and has been studied by various authors (see, e.g., $[17,30,31]$ and references therein).

The above analysis can be easily extended to $N=1$ supergravity with a cosmological constant. When the spinor field obeys the boundary conditions (V.1), there is no divergent term proportional to $\delta R$ in the variation of the supersymmetry generator. There is, however, a finite piece which cannot be integrated away and which must then be compensated by an appropriate additional gauge transformation. The notion of a global supersymmetry transformation at infinity has thus also to be "improved" when the cosmological constant is varied.

The fact that one can still have a well-defined variational principle when the cosmological constant is varied enables one to compare the various vacuum antide Sitter spaces with different radii of curvature. These (supersymmetric) solutions appear here as different states of the same system possessing classically the same energy, namely zero, because the energy is the same functional as when the cosmological constant is unvaried.

It is important to understand clearly that this conclusion is not just a conventional adjustment of a "zero-point" energy. Indeed, the most one can do by adjusting the zero of energy is to make the energy to vanish for one value of $\Lambda$. After this is done one can then evaluate the energy for other values of $\Lambda$. According to our analysis that energy turns out to be also zero. This result, although simple, is not empty since according to previous ideas there was no way to compare these different vacua.

The case $\Lambda=0$ (asymptotically flat space) deserves special consideration. From a mathematical point of view our analysis of the surface integral applies only to $\Lambda \neq 0$. However, by continuity, this limiting case should also have zero energy. This conclusion may be also arrived at by noticing that the surface integrals take upon contraction $(R \rightarrow \infty)$ the ADM form whose value is zero for Minkowski space.

\section{Appendix A. Killing Vectors, Killing Spinors, OSp(1,4) Algebra}

The anti-de Sitter metric with radius of curvature $R$ can be written as

$$
d \grave{s}^{2}=-\left[1+\varrho^{2}\right] d t^{2}+\left[1+\varrho^{2}\right]^{-1} d r^{2}+r^{2} d \Omega^{2}
$$

with $\varrho=r R^{-1}$. It is the solution to the vacuum Einstein equations (with cosmological constant $\Lambda=-3 / R^{2}$ ) that possesses the maximum number of 
isometries. In the above coordinate system, its ten Killing vectors $U_{a b}=-U_{b a}$ $(a, b=1, \ldots, 5) \mathrm{read}$

$$
\begin{aligned}
U_{51}= & R\left(1+\varrho^{2}\right)^{1 / 2} \mathbf{n}, \\
U_{21}= & -R \varrho \sin \tau \sin \theta \cos \phi \mathbf{n}+\left(1+\varrho^{2}\right)^{1 / 2} \cos \tau \sin \theta \cos \phi \frac{\partial}{\partial \varrho} \\
& +\varrho^{-1}\left(1+\varrho^{2}\right)^{1 / 2} \cos \tau\left(\cos \theta \cos \phi \frac{\partial}{\partial \theta}-\frac{\sin \phi}{\sin \theta} \frac{\partial}{\partial \phi}\right) \\
U_{31}= & -\varrho R \sin \tau \sin \theta \sin \phi \mathbf{n}+\left(1+\varrho^{2}\right)^{1 / 2} \cos \tau \sin \theta \sin \phi \frac{\partial}{\partial \varrho} \\
& +\varrho^{-1}\left(1+\varrho^{2}\right)^{1 / 2} \cos \tau\left(\cos \theta \sin \phi \frac{\partial}{\partial \theta}+\frac{\cos \phi}{\sin \theta} \frac{\partial}{\partial \phi}\right) \\
U_{41}= & -\varrho R \sin \tau \cos \theta \mathbf{n}+\left(1+\varrho^{2}\right)^{1 / 2} \cos \tau \cos \theta \frac{\partial}{\partial \varrho} \\
& -\varrho^{-1}\left(1+\varrho^{2}\right)^{1 / 2} \cos \tau \sin \theta \frac{\partial}{\partial \theta}, \\
U_{25}= & R \varrho \cos \tau \sin \theta \cos \phi \mathbf{n}+\left(1+\varrho^{2}\right)^{1 / 2} \sin \tau \sin \theta \cos \phi \frac{\partial}{\partial \varrho} \\
& +\varrho^{-1}\left(1+\varrho^{2}\right)^{1 / 2} \sin \tau\left(\cos \theta \cos \phi \frac{\partial}{\partial \theta}-\frac{\sin \phi}{\sin \theta} \frac{\partial}{\partial \phi}\right) \\
U_{35}= & R \varrho \cos \tau \sin \theta \sin \phi \mathbf{n}+\left(1+\varrho^{2}\right)^{1 / 2} \sin \tau \sin \theta \sin \phi \frac{\partial}{\partial \varrho} \\
& +\varrho^{-1}\left(1+\varrho^{2}\right)^{1 / 2} \sin \tau\left(\cos \theta \sin \phi \frac{\partial}{\partial \theta}+\frac{\cos \phi}{\sin \theta} \frac{\partial}{\partial \phi}\right), \\
U_{34}= & -\sin \phi \frac{\partial}{\partial \theta}-\operatorname{cotg} \theta \cos \phi \frac{\partial}{\partial \phi}, \\
U_{42}= & \cos \phi \frac{\partial}{\partial \theta}-\operatorname{cotg} \theta \sin \phi \frac{\partial}{\partial \phi} . \\
U_{45}= & R \varrho \cos \tau \cos \theta \mathbf{n}+\left(1+\varrho^{2}\right)^{1 / 2} \sin \tau \cos \theta \frac{\partial}{\partial \varrho} \\
& -\varrho^{-1}\left(1+\varrho^{2}\right)^{1 / 2} \sin \tau \sin \theta \frac{\partial}{\partial \theta}, \\
& ,
\end{aligned}
$$

Here, $\tau=t R^{-1}$ and $\mathbf{n}$ is the unit normal to the hypersurfaces $t=\mathrm{const}$,

$$
\mathbf{n}=\left(1+\varrho^{2}\right)^{-1 / 2} \frac{\partial}{\partial t}
$$


$U_{51}$ is everywhere timelike and defines $R$ times the "energy". Upon contraction $(R \rightarrow \infty)$ (A.1) becomes the Minkowski metric, $R^{-1} U_{51}$ becomes the generator of time translations in flat space, $R^{-1} U_{21}, R^{-1} U_{31}$, and $R^{-1} U_{41}$ become the generators of space translations whereas the other Killing vectors keep their meaning as generators of boosts $\left(U_{15}, U_{25}, U_{35}\right)$ and of spatial rotations $\left(U_{23}\right.$, $\left.U_{31}, U_{41}\right)$.

The Killing vector fields obey the algebra of $\mathrm{O}(3,2)$ (in the Lie bracket),

$$
\left[U_{a b}, U_{c d}\right]=C^{e f}{ }_{a b c d} U_{e f}
$$

with

$$
C^{e f}{ }_{a b c d}=\frac{1}{2} \eta_{b c}\left(\delta_{a}^{e} \delta_{d}^{f}-\delta_{a}^{f} \delta_{d}^{e}\right)-\frac{1}{2} \eta_{b d}\left(\delta_{a}^{e} \delta_{c}^{f}-\delta_{a}^{f} \delta_{c}^{e}\right)-(a \leftrightarrow b) .
$$

The signature of the five-dimensional metric $\eta_{a b}$ is $(-,+,+,+,-)$.

They are two Casimir invariants for $\mathrm{O}(3,2)$. The first one is quadratic

$$
I_{1}=\frac{1}{2} J_{a b} J^{a b} \text {. }
$$

The other one is of fourth order

$$
I_{2}=\frac{1}{2} J_{a}^{b} J_{b}^{c} J_{c}^{d} J_{d}^{a}-\frac{1}{4}\left(J_{a}^{b} J_{b}{ }^{a}\right)^{2} .
$$

( $J_{a b}$ are elements of the Lie algebra.) Upon contraction, $I_{1} / R^{2}$ becomes the mass squared and $-I_{2} / 2 R^{2}$ the square of the norm of the Pauli-Lubanski vector $W_{\mu}=(1 / 2) \varepsilon_{\mu \alpha \beta \gamma} P^{\alpha} J^{\beta \gamma}$.

In order to study the invariance of the boundary conditions put forward in the main text and the convergence of the surface integrals for $J_{a b}$, one needs the asymptotic behavior of the Killing vector components. Straightforward analysis of (A.2-5) reveals that

$$
\begin{gathered}
\xi^{t}=O(1), \quad \xi^{\theta}=O(1), \quad \xi^{\phi}=O(1), \\
\xi_{, r}^{t}=O\left(r^{-3}\right), \quad \xi_{, r}^{\theta}=O\left(r^{-3}\right), \quad \xi_{, r}^{\phi}=O\left(r^{-3}\right), \\
\xi^{r}=O(r), \quad \xi_{, r}^{r}=O(1),
\end{gathered}
$$

and

$$
\xi^{\perp}=O(r)
$$

Anti-de Sitter space possesses also four linearly independent Killing spinors $U_{B}$ which obey by definition

$$
\nabla_{\mu} U_{A}=\frac{1}{2 R} \gamma_{\mu} U_{A}
$$

$(A=1,2,3,4)$. These Killing spinors play an important role in the theory because they define supersymmetry transformations which leave the spin $3 / 2$ field $\psi_{\mu}$ asymptotically invariant [5] $\left(\delta \psi_{\mu}=\nabla_{\mu} \varepsilon-(2 R)^{-1} \gamma_{\mu} \varepsilon\right.$ [27] vanishes at infinity). [ $\nabla_{\mu}$ is here the spinor covariant derivative without torsion. The Dirac $\gamma$-matrices are taken to be real, and so are the spinor fields ("Majorana spinors"). $\gamma^{0}$ is antisymmetric whereas the $\gamma^{k}$ are symmetric.] 
The general solution to (A.9) is

$$
\begin{aligned}
U= & {\left[\left(\frac{\left(1+\varrho^{2}\right)^{1 / 2}+1}{2}\right)^{1 / 2}+\left(\frac{\left(1+\varrho^{2}\right)^{1 / 2}-1}{2}\right)^{1 / 2} \gamma_{(1)}\right] } \\
& \cdot\left(\cos \frac{\theta}{2}+\sin \frac{\theta}{2} \gamma_{(1)} \gamma_{(2)}\right)\left(\cos \frac{\phi}{2}+\sin \frac{\phi}{2} \gamma_{(2)} \gamma_{(3)}\right) \\
& \cdot\left(\cos \frac{\tau}{2}-\sin \frac{\tau}{2} \gamma^{(0)}\right) \dot{U},
\end{aligned}
$$

where $\stackrel{U}{ }$ is a spinor whose components are constant in the orthonormal frame $\omega^{\mu}$ in which we carry out the calculations [see (II.8)]. Different choices of that constant spinor define the different $U_{A}$. For definiteness we take here $\left(\dot{U}_{A}\right)^{B}$ $=\delta_{A}{ }^{B} R^{1 / 2}$.

The solution (A.10) is double-valued (e.g., it changes sign under $\phi \rightarrow \phi+2 \pi$ ). This, however, simply results from the fact that the frame $\omega^{\mu}$ rotates by $2 \pi$ with respect to the standard frames that define the spinor structure when $\phi \rightarrow \phi+2 \pi$, and is analogous to what happens in flat space when one expresses spinors in spherical coordinates.

The Killing spinors possess a number of interesting properties:

(i) They grow as $\varrho^{1 / 2}$ at infinity. Moreover, their leading part is given by $\varrho^{1 / 2}\left(1+\gamma_{(1)}\right)$ [where (1) is the radial leg of the tetrad] acting on some $\varrho$-independent spinor and hence, they belong to the eigenspace of $\gamma_{(1)}$ with eigenvalue -1 to that order. The next order $\left(\varrho^{-1 / 2}\right)$ belongs to the other eigenspace, the one with eigenvalue +1 .

(ii) They are such that

$$
\bar{U}_{A} \gamma^{\mu} U_{B}=\frac{1}{2} M_{A B}{ }^{a b} U_{a b}^{\mu},
$$

where the 10 matrices $M^{a b}$ are given by

$$
M^{a b}=-M^{b a}, \quad M^{a b}=-\gamma^{0} \Gamma^{a} \Gamma^{b}, \quad a>b,
$$

with

$$
\Gamma^{1}=-I, \quad \Gamma^{2}=\gamma^{2}, \quad \Gamma^{3}=\gamma^{3}, \quad \Gamma^{4}=\gamma^{1}, \quad \Gamma^{5}=\gamma^{0} .
$$

(iii) The Lie derivatives of the Killing spinors along the Killing vector fields are

$$
\mathfrak{£}_{U_{a b}} U_{A}=U_{B}\left(N_{a b}\right)_{B A},
$$

where the matrices $N_{a b}$ read explicitly

$$
\begin{gathered}
N_{a b}=-N_{b a}, \quad N_{a b}=\Gamma_{a} \Gamma_{b}, \quad a>b, \\
\Gamma_{a}=\eta_{a b} \Gamma^{b}, \quad N_{a b}=\gamma^{0} M_{a b} .
\end{gathered}
$$

(For a definition of Lie derivatives of spinors, see $[33,34]$ and references therein.)

The set of Killing vectors-Killing spinors of anti-de Sitter space is thus closed under the operations (A.6), (A.11), and (A.13). Those relations define the $\operatorname{OSp}(1,4)$ "superalgebra." The left hand side of Eq. (A.11) may be thought of as defining the "Lie derivative of the spinor $U_{B}$ along the spinor $U_{A}$." It yields a result which is 
symmetric in $A$ and $B$ and is a vector. The "Lie derivative of $U_{a b}$ along $U_{A}$ " may in turn be defined to be the negative of (A.13) and is thus a spinor. The combination $\left(U_{a b}, U_{A}\right)$ may be regarded as a single geometrical object for each value of $a b, A$. The whole set of equations may then be described as giving the "Lie-Derivative" of each of these objects along each other.

\section{Appendix B. The Kerr-Anti-de Sitter Metric}

The solution of Einstein equations with mass, angular momentum and a (negative) cosmological constant has been worked out in [3]. The metric is explicitly given by

$$
\begin{aligned}
d s^{2}= & -\left(1-\frac{2 m \hat{r}}{\hat{r}^{2}+a^{2} \cos ^{2} \hat{\theta}}+\frac{\hat{r}^{2}+a^{2} \sin ^{2} \hat{\theta}}{R^{2}}\right) d \hat{t}^{2} \\
& -2 a \sin ^{2} \hat{\theta}\left(\frac{2 m \hat{r}}{\hat{r}^{2}+a^{2} \cos ^{2} \hat{\theta}}-\frac{\hat{r}^{2}+a^{2}}{R^{2}}\right) d \hat{d} d \hat{\phi} \\
& +\left(\hat{r}^{2}+a^{2} \cos ^{2} \hat{\theta}\right)\left[\frac{d \hat{r}^{2}}{\left(\hat{r}^{2}+a^{2}\right)(\hat{r} / R)^{2}+\hat{r}^{2}-2 m \hat{r}+a^{2}}+\frac{d \hat{\theta}^{2}}{1-\left(\frac{a}{R}\right)^{2} \cos ^{2} \hat{\theta}}\right] \\
& +\sin ^{2} \hat{\theta}\left[\frac{2 m \hat{r} a^{2} \sin ^{2} \hat{\theta}}{\hat{r}^{2}+a^{2} \cos ^{2} \hat{\theta}}+\left(\hat{r}^{2}+a^{2}\right)\left(1-\frac{a^{2}}{R^{2}}\right)\right] d \hat{\phi}^{2} .
\end{aligned}
$$

The parameter $a$, which will be related to the angular momentum per unit mass, obeys $|a|<R$.

When $a \neq 0$, the metric (B.1) does not obey the boundary conditions of the text. A coordinate transformation is necessary to bring it to a manifestly asymptotically anti-de Sitter form.

This coordinate transformation is easily found by noticing that the metric (B.1) with $m=0$ is the anti-de Sitter solution written in unusual coordinates. The coordinate transformation implicitly defined by

$$
\begin{gathered}
\phi=\left[1-\left(\frac{a}{R}\right)^{2}\right] \hat{\phi}+\frac{a}{R^{2}} \hat{t}, \quad t=\hat{t}, \\
r \cos \theta=\hat{r} \cos \hat{\theta}, \\
{\left[1-\left(\frac{a}{R}\right)^{2}\right] r^{2}=\hat{r}^{2}+a^{2} \sin ^{2} \hat{\theta}-\left(\frac{a}{R}\right)^{2} \hat{r}^{2} \cos ^{2} \hat{\theta},}
\end{gathered}
$$

indeed brings (B.1) with $m=0$ into the standard anti-de Sitter form. It turns out to be the desired transformation in the general case $m \neq 0$.

In order to compute $J_{a b}$, we need the deviation from the anti-de Sitter background

$$
\begin{gathered}
h_{\hat{t} \hat{t}}=\frac{2 m \hat{r}}{\hat{r}^{2}+a^{2} \cos ^{2} \hat{\theta}}, \\
h_{\hat{t} \hat{\phi}}=-\frac{2 a m \sin ^{2} \hat{\theta} \hat{r}}{\hat{r}^{2}+a^{2} \cos ^{2} \hat{\theta}},
\end{gathered}
$$




$$
\begin{gathered}
h_{\hat{r} \hat{r}}=\frac{\left(\hat{r}^{2}+a^{2} \cos ^{2} \hat{\theta}\right)}{\left(\hat{r}^{2}+a^{2}\right)\left[(\hat{r} / R)^{2}+1\right]} \frac{2 m \hat{r}}{\left[\left(\hat{r}^{2}+a^{2}\right)(\hat{r} / R)^{2}+\hat{r}^{2}-2 m \hat{r}+a^{2}\right]}, \\
h_{\hat{\phi} \hat{\phi}}=\frac{2 m \hat{r} a^{2} \sin ^{4} \hat{\theta}}{\hat{r}^{2}+a^{2} \cos ^{2} \hat{\theta}}
\end{gathered}
$$

in the new coordinate system only to the leading order in $1 / r$.

Straightforward computation yields as non-vanishing components $h_{\lambda \mu}$

$$
\begin{gathered}
h_{t t}=\frac{2 m}{r}\left(1-\alpha^{2} \sin ^{2} \theta\right)^{-5 / 2}+O\left(r^{-3}\right), \\
h_{t \phi}=-\frac{2 a m \sin ^{2} \theta}{r}\left(1-\alpha^{2} \sin ^{2} \theta\right)^{-5 / 2}+O\left(r^{-3}\right), \\
h_{\phi \phi}=\frac{2 m a^{2} \sin ^{4} \theta}{r}\left(1-\alpha^{2} \sin ^{2} \theta\right)^{-5 / 2}+O\left(r^{-3}\right), \\
h_{r r}=\frac{2 m R^{4}}{r^{5}}\left(1-\alpha^{2} \sin ^{2} \theta\right)^{-3 / 2}+O\left(r^{-7}\right), \\
h_{\theta r}=-\frac{2 m R^{2} a^{2}}{r^{4}}\left(1-\alpha^{2} \sin ^{2} \theta\right)^{-5 / 2} \sin \theta \cos \theta+O\left(r^{-6}\right), \\
h_{\theta \theta}=\frac{2 m a^{4}}{r^{3}}\left(1-\alpha^{2} \sin ^{2} \theta\right)^{-7 / 2} \sin ^{2} \theta \cos ^{2} \theta+O\left(r^{-5}\right),
\end{gathered}
$$

with $\alpha=a / R$.

The extrinsic curvature of the surfaces $t=$ const is easily computed from the expression $K_{i j}=(2 N)^{-1}\left(-g_{i j, 0}+N_{i \mid j}+N_{j \mid i}\right)$. It is given by

$$
\begin{gathered}
K_{r \theta}=0, \\
K_{r \phi}=\frac{3 a m R \sin ^{2} \theta}{r^{3}}\left(1-\alpha^{2} \sin ^{2} \theta\right)^{-5 / 2}+O\left(r^{-5}\right), \\
K_{\theta \phi}=-\frac{5 \alpha a^{2} m}{r^{2}} \sin ^{3} \theta \cos \theta\left(1-\alpha^{2} \sin ^{2} \theta\right)^{-7 / 2}+O\left(r^{-4}\right), \\
K_{r r}=K_{\theta \theta}=K_{\phi \phi}=0
\end{gathered}
$$

Its trace is zero. The gravitational momentum $\pi^{i j}$ has consequently the following non-vanishing components

$$
\begin{gathered}
\pi^{r \phi}=-\frac{3 a m \sin \theta}{r^{2}}\left(1-\alpha^{2} \sin ^{2} \theta\right)^{-5 / 2}+O\left(r^{-4}\right), \\
\pi^{\theta \phi}=O\left(r^{-5}\right) .
\end{gathered}
$$

We are now in a position to evaluate the surface integrals $J_{a b}$. Because the gravitational variables are $\phi$-independent, one immediately infers that $J_{21}=J_{31}$ $=J_{25}=J_{35}=J_{34}=J_{42}=0\left(\int_{0}^{2 \pi} \cos \phi d \phi=\int_{0}^{2 \pi} \sin \phi d \phi=0\right)$. Hence, only energy, 
angular momentum about the axis of symmetry ("the $z$-axis") as well as the two types of boost generators along the $z$-axis can have a non-zero value.

Let us consider $J_{41}$ first ("boost along $z$ "). Since the gravitational variables are invariant for $\theta \rightarrow \pi-\theta$ and since $U_{41}^{\perp}$ and $U_{41}^{r}$ both contain an odd power of $\cos \theta$, the integral (III.7) reduces to the $-\oint d^{2} S_{r} \stackrel{\circ}{G}^{r \theta k l} U_{a b, \theta}^{\perp} h_{k l}$ and to the $2 \oint d^{2} S_{r} U_{a b}^{\theta} \pi_{\theta}^{r}$ pieces. But these are zero in the limit $r \rightarrow \infty\left[h_{\theta r}=O\left(r^{-4}\right)\right.$ and $\left.\pi_{\theta}{ }^{r}=0\right]$, so that $J_{41}$ vanishes. Similarly, $J_{45}$ is equal to zero by the same reasoning. Hence, we are left with $J_{51}$ (energy) and $J_{23}$ (angular momentum about the axis of symmetry).

These charges do not vanish. Straightforward use of the formula (III.7) leads to

$$
\begin{aligned}
J_{51}= & 8 \pi m R \int_{0}^{\pi} \sin \theta\left(1-\alpha^{2} \sin ^{2} \theta\right)^{-3 / 2} \\
& +\frac{12 \pi m a^{2}}{R} \int_{0}^{\pi} \sin ^{3} \theta\left(1-\alpha^{2} \sin ^{2} \theta\right)^{-5 / 2} \\
= & \frac{16 \pi m R}{\left(1-\alpha^{2}\right)^{2}}
\end{aligned}
$$

and

$$
\begin{aligned}
J_{23} & =-12 \pi a m \int_{0}^{\pi} \sin ^{3} \theta\left(1-\alpha^{2} \sin ^{2} \theta\right)^{-5 / 2} \\
& =-\frac{16 \pi a m}{\left(1-\alpha^{2}\right)^{2}} .
\end{aligned}
$$

In the limit $R \rightarrow \infty, J_{51} / R$ and $J_{23}$ tend to their $\Lambda=0$ value.

The above computation shows that $m$ cannot be interpreted as the mass (although it is closely related to it). Rather, the mass is given by

$$
m^{\prime}=\frac{m}{\left(1-\alpha^{2}\right)}
$$

whereas the angular momentum $a^{\prime}$ per unit mass is just

$$
a^{\prime}=\frac{J_{23}}{R J_{51}}=-a
$$

Note that for a given $m, m^{\prime} \rightarrow \infty$ as $a$ approaches $R$.

\section{Appendix C. Asymptotic Isometries}

In this appendix the "asymptotic Killing equations"

$$
£_{\xi} g_{\lambda \mu}=O\left(r^{-k_{\lambda \mu}}\right)
$$

are considered (here, $k_{t t}=k_{t \theta}=k_{t \phi}=k_{\theta \theta}=k_{\theta \phi}=k_{\phi \phi}=1, k_{t r}=k_{\theta r}=k_{\phi r}=4, k_{r r}=5-$ see Sect. II). These equations define coordinate transformations which preserve the asymptotic form of the metric, i.e., asymptotic isometries. 
It turns out that any solution of the Eq. (C.1) for the background metric

$$
\mathfrak{f}_{\xi} \stackrel{\circ}{\partial \mu}_{\lambda \mu}=O\left(r^{-k_{\lambda \mu}}\right) \text {, }
$$

is also a solution of (C.1). Accordingly, it is enough to solve the asymptotic Killing Equations (C.2).

Straightforward computations show that (C.2) is satisfied if and only if $\xi^{\lambda}$ differs from a Killing vector field $\frac{1}{2} \xi_{\infty}^{a b} U_{a b}^{\mu}$ by terms of the following order:

$$
\begin{aligned}
\xi^{0}-\frac{1}{2} \xi_{\infty}^{a b} U_{a b}^{0} & =O\left(r^{-5}\right), \\
\text { (4) } \xi^{r}-\frac{1}{2} \xi_{\infty}^{a b} U_{a b}^{r} & =O\left(r^{-2}\right), \\
\text { (4) } \xi^{\theta}-\frac{1}{2} \xi_{\infty}^{a b} U_{a b}^{\theta} & =O\left(r^{-5}\right), \\
\text { (4) } \xi^{\phi}-\frac{1}{2} \xi_{\infty}^{a b} U_{a b}^{\phi} & =O\left(r^{-5}\right) .
\end{aligned}
$$

[Here the upper left index ${ }^{(4)}$ emphasizes that one is dealing with spacetime contravariant components.] Since the right-hand sides of (C.3a-d) go to zero at infinity, they do not contribute to the surface integrals $J_{a b}$. Only the asymptotic anti-de Sitter part contributes, which means that the anti-de Sitter group is the asymptotic symmetry group.

In the case of $N=1$ supergravity, one considers besides (C.1) supersymmetry transformations which leave the fields asymptotically invariant. Again, it is enough to study the asymptotic Killing-spinor equations for the empty anti-de Sitter background, where they read

$$
\begin{aligned}
& {\stackrel{\circ}{\nabla_{\hat{\lambda}}} \varepsilon}=r^{-3 / 2}\left(1-\gamma_{(1)}\right) u_{\hat{\lambda}}(t, \theta, \phi)+O\left(r^{-5 / 2}\right), \\
& {\stackrel{\circ}{\nabla_{r}} \varepsilon}^{-}=r^{-7 / 2}\left(1+\gamma_{(1)}\right) u_{r}(t, \theta, \phi)+O\left(r^{-9 / 2}\right) .
\end{aligned}
$$

Equations (C.4) easily yield

$$
\varepsilon=\varepsilon^{A} U_{A}+r^{-5 / 2}\left(1+\gamma_{(1)}\right) a(t, \theta, \phi)+O\left(r^{-7 / 2}\right),
$$

where $a$ is an arbitrary $r$-independent spinor and where the $\varepsilon^{A}$ are constants.

Again, the difference between $\varepsilon$ and a true Killing spinor has no effect on the surface integrals $Q_{A}$ and $\operatorname{OSp}(1,4)$ is therefore the asymptotic symmetry superalgebra.

Let us finally point out that $\psi_{r}$ can be set equal to zero by a suitable choice of the proper gauge piece of (C.5) (which is obtained by setting the constant $\varepsilon^{A}$ equal to zero). This result is related to the fact that $\psi_{r}$ drops out from the supercharges $Q_{A}$. However, taking $\psi_{r}=0$ does not really simplify the discussion and this gauge condition has not been adopted here. A similar feature arises when the cosmological constant vanishes [35].

[We would like to point out here that when $\Lambda=0$ the asymptotic superalgebra is infinite dimensional due to the angle dependent translations (which are absent when $\Lambda<0$ ) but its fermionic component is nevertheless just the usual "square root" of the Poincaré algebra and is thus four-dimensional. Spinor fields which behave like $\varepsilon(t, \theta, \phi)$ (in the cartesian frames) and which yield angledependent translations when squared must be discarded because their covariant derivatives only fall off as $1 / r$ at infinity and hence, do not preserve the flat space boundary conditions $\psi_{i} \sim 1 / r^{2}$.] 


\section{Appendix D. Equivalence of Our Boundary Conditions with the Boundary Conditions on the Conformal Completion of Spacetime}

We have shown in the main text that our boundary conditions for asymptotically anti-de Sitter spaces imply the ones proposed in [4]. We prove here the converse, namely, that those of [4] imply ours.

We begin the analysis by assuming, as in [4], that one can conformally rescale the "physical" metric $d s^{2}$,

$$
d \bar{s}^{2}=\Omega^{2} d s^{2}
$$

so that the surface at infinity $I$, which is located where the conformal factor $\Omega$ vanishes, is brought in to a finite distance in the new metric. We also assume, for simplicity, that the physical metric obeys exactly the vacuum Einstein equations everywhere [although, as will be apparent in the sequel, one only needs these vacuum equations - equations (D.4) below - to hold asymptotically to order $\Omega$ as in [4] - to prove the equivalence].

Lastly, in order to simplify the mathematical discussion as much as possible, we also take the "unphysical" metric $\bar{g}_{\alpha \beta}$ to be analytic close to and on the surface at infinity. We will not go in this paper into the problem of relaxing this condition - something that can clearly be done.

The Ricci tensors of two metrics which are conformal to each other according to (D.1) are related by

$$
R_{\alpha \beta}=\bar{R}_{\alpha \beta}+2 \frac{\Omega_{; \alpha \beta}}{\Omega}+\bar{g}_{\alpha \beta} \frac{\Delta_{2} \Omega}{\Omega}-3 \bar{g}_{\alpha \beta} \frac{\Delta_{1} \Omega}{\Omega^{2}}
$$

(see e.g. [36]). Here and everywhere in this appendix, the semi-column denotes the covariant derivative in the "unphysical" metric $\bar{g}_{\alpha \beta}$, whereas $\Delta_{2} \Omega$ and $\Delta_{1} \Omega$ stand for

$$
\Delta_{2} \Omega=\bar{g}^{\alpha \beta} \Omega_{; \alpha \beta}, \quad \Delta_{1} \Omega=\bar{g}^{\alpha \beta} \Omega_{; \alpha} \Omega_{; \beta},
$$

respectively. Besides, we refer the components of tensors to frames which are smooth in the vicinity of $I$ [unlike the orthonormal frame (II.8) of Sect. II].

The metric $\bar{g}_{\alpha \beta}$ and the function $\Omega$ are by hypothesis regular close to and on the surface at infinity. Accordingly, the Ricci tensor $\bar{R}_{\alpha \beta}$ and the covariant derivatives $\Omega_{; \alpha \beta}, \Omega_{; \alpha}$ are also regular. It follows from this remark and the Einstein equations for the physical metric,

$$
R_{\alpha \beta}=-3 g_{\alpha \beta}=-3 \frac{\bar{g}_{\alpha \beta}}{\Omega^{2}},
$$

that the square of the gradient of $\Omega$ must be constant and equal to one on the surface at infinity $\Omega=0$. This results from the fact that the most singular parts ( $\Omega^{-2}$ terms) on both sides of the identity (D.2) must be equal. Here, we have assumed, for definiteness, that the radius of curvature is itself unity. There is no loss of generality in doing so, because if one can prove the equivalence of both sets of boundary conditions for a given radius of curvature, then, equivalence for all radii follows upon constant conformal rescaling of the physical metric. 
A useful simplification is introduced by noticing that the unphysical metric $d \bar{s}^{2}$ is not uniquely determined by the physical metric $d s^{2}$, since one still has the freedom of making conformal transformations

$$
d \bar{s}^{2}=\omega^{2} d \bar{s}^{2}
$$

with a function $\omega$ which vanishes nowhere. Under that rescaling, the conformal factor becomes

$$
\Omega^{\prime}=\omega \Omega .
$$

One can take advantage of this freedom to make the gradient of the new conformal factor (D.6), computed in the new unphysical metric (D.5), equal to one not only on the surface at infinity $I$, but also in its vicinity,

$$
\Delta_{1}^{\prime} \Omega^{\prime}=1 \text {. }
$$

Equation (D.7) can be read as a partial differential equation for $\omega$ and possesses locally a solution.

Furthermore, one may exploit still further the freedom (D.6) by requiring in addition that the induced metric on $I$ be given by

$$
\left.d \bar{s}^{2}\right|_{I}=-d t^{2}+d \theta^{2}+\sin ^{2} \theta d \phi^{2} .
$$

This can always be done as a result of Hawking's demand that the conformal group of motions of $I$ be the anti-de Sitter group [4]. [The ten $\mathrm{O}(3,2)$ conformal Killing vectors of (D.8) are simply obtained from the vectors (A.5) by subtracting their $\partial / \partial \varrho$-component.]

We will assume from now on that the conformal factor has been chosen according to (D.7) and (D.8) and we will drop the prime on the new unphysical metric.

In order to make contact with the boundary conditions put forward in Sect. II, we first construct an appropriate coordinate system in the neighborhood of the surface $\Omega=0$ as follows. The first coordinate $u$ is taken to be just $\Omega$ itself. The other coordinates $y^{a}(a=1,2,3)$ are, on $u=0$, the coordinates $t, \theta, \phi$ of (D.8). They are propagated off the surface at infinity in such a way that the curves of constant $t, \theta, \phi$ are orthogonal to the surfaces $u=$ const.

Because the gradient of $\Omega$ has unit magnitude, this coordinate system turns out to be gaussian,

$$
d \bar{s}^{2}=d u^{2}+\bar{g}_{a b}(u, y) d y^{a} d y^{b},
$$

with $\bar{g}_{a b}$ given by (D.8) on $u=0$.

Next, we investigate the consequences of Einstein Eq. (D.4) on the metric functions $\bar{g}_{a b}$. Let

$$
\bar{g}_{a b}(u, y)=\sum_{n=0} \frac{1}{n !} \stackrel{(n)}{g}_{a b}(y) u^{n},
$$

be the Taylor expansion of $\bar{g}_{a b}$. From (D.8), the non vanishing coefficients $\stackrel{(o)}{\bar{g}}_{a b}(y)$ are equal to

$$
\stackrel{o}{\bar{g}}_{t t}=-1, \quad \stackrel{o}{g}_{\theta \theta}=1, \quad \stackrel{o}{\bar{g}}_{\phi \phi}=\sin ^{2} \theta .
$$


In view of (D.7), the vacuum Einstein equations (D.4) for the physical metric become, after some elementary rearrangements,

$$
A_{\alpha \beta} \equiv \Omega \bar{G}_{\alpha \beta}+2 \Omega_{; \alpha \beta}-2 \Delta_{2} \Omega \bar{g}_{\alpha \beta}=0 \text {. }
$$

Here $\bar{G}_{\alpha \beta}$ is the Einstein tensor of $\bar{g}_{\alpha \beta}$. Note that these equations are not the vacuum Einstein equations for the metric $\bar{g}_{\alpha \beta}$. Nevertheless, they possess some of their general features.

For instance, because of the contracted Bianchi identity for $\bar{G}_{\alpha \beta}$, the Eq. (D.12) are not all independent. It actually turns out that the "constraint equations"

$$
A_{u u}=0, \quad A_{u a}=0,
$$

hold for all values of the "time" $u$ as a consequence of the "dynamical equations"

$$
A_{a b}=0
$$

provided (i) that the second order term $\stackrel{(2)}{A}_{u u}$ in the Taylor expansion of $A_{u u}$ in powers of $u$ is zero,

$$
\stackrel{(2)}{A}=0
$$

and (ii), that the $u^{3}$-coefficient $\stackrel{(3)}{A}_{a u}$ in the expansion of $A_{a u}$ vanishes too,

$$
\stackrel{(3)}{A}_{a u}=0 \text {. }
$$

The detailed proof of this statement is straightforward and is based (i) on the contracted Bianchi identity for $\bar{G}_{\alpha \beta}$ as mentioned above; and (ii) on the Taylor expansion of the Eq. (D.12). It will not be reproduced here.

It follows from the above analysis that in order to solve the system (D.12), it is sufficient to consider the Eqs. (D.14), (D.15), and (D.16).

Let us first study the dynamical Eq. (D.14), which contain the first and second derivatives of $\bar{g}_{a b}$.

Straightforward computations reveal that to order one, these equations imply

$$
{\stackrel{(1)}{\bar{g}_{a b}}}=0 \text {. }
$$

Relation (D.17) is equivalent to the statement that the extrinsic curvature of the surface at infinity vanishes since $\stackrel{(1)}{g}_{a b}$ reduces there to the first derivative of the metric with respect to $u$ and since the "shift" $g_{a u}$ vanishes.

Next, lengthy - but still elementary - calculations show that to order $u$, the Eq. (D.12) determine completely $\stackrel{(2)}{g}_{a b}$ as

$$
\begin{aligned}
& \stackrel{(2)}{\bar{g}}_{t t}=-1, \\
& \stackrel{(2)}{\bar{g}}_{\theta \theta}=-1, \\
& \stackrel{(2)}{\bar{g}}_{\phi \phi}=-\sin ^{2} \theta .
\end{aligned}
$$


The conditions (D.17) and (D.18) are already sufficient to guarantee that the physical metric $g_{\alpha \beta}$ possesses the asymptotic behavior of Sect. II. This can be explicitly seen by making the change of radial variables

$$
r=\frac{1}{u}-\frac{u}{4}
$$

which transforms the metric $d s^{2}=\Omega^{-2} d \bar{s}^{2}$ into

$$
d s^{2}=d s_{a d S}^{2}+h_{\alpha \beta} d x^{\alpha} d x^{\beta} .
$$

Here, $d s_{a d S}^{2}$ is the anti-de Sitter metric written in the standard coordinate system (II.1) whereas the perturbation $h_{\alpha \beta}$ has the asymptotic decrease dictated by (II.2).

Although this completes the proof of the equivalence between Hawking boundary conditions and ours, it is of interest to study further the Eq. (D.12), if only to see "how many" non trivial solutions $g_{\alpha \beta}$ of Einstein equations exist which are asymptotically anti-de Sitter. This is done by analyzing the next order terms in the expansion of $A_{a b}$ (orders $u^{2}$ and beyond). That analysis is devoided of conceptual difficulties and we only report the results here.

To order $u^{2}$, the dynamical Eq. (D.14) completely degenerate and are identically fulfilled as a consequence of (D.17). Accordingly, they yield no restriction on $\stackrel{(3)}{\bar{g}}_{a b}$, which can be chosen arbitrarily at this stage. In contrast, the higher orders $\left(u^{3}, u^{4}, \ldots\right)$ of the dynamical equations completely determine the next orders of $\bar{g}_{a b}\left(\right.$ coefficients $\stackrel{(4)}{\bar{g}}_{a b}$ and higher $)$ in terms of $\stackrel{(3)}{g}_{a b}$. Hence the general solution of the dynamical Eq. (D.14) contains six functions

$$
\stackrel{(3)}{\bar{g}}_{a b} \equiv p_{a b}
$$

of the three variables $t, \theta, \phi$.

It remains to impose the constraint Eq. (D.15) and (D.16). These turn out to imply restrictions on the "initial data" $p_{a b}$ which explicitly read

$$
p_{a b} \stackrel{o}{a}^{a b}=0
$$

and

$$
\left.p_{a}^{b}\right|_{b}=0
$$

In (D.23), the covariant derivative is computed with the metric (D.8). The linear conditions (D.22), (D.23) are the only constraints on the symmetric tensor $p_{a b}$.

The conclusion of the study of the Eq. (D.12) is that there are as many asymptotically anti-de Sitter solutions of the Einstein equations with a negative cosmological constant as there are symmetric, traceless and transverse second rank tensors on the manifold $R \times S^{2}$ endowed with the metric (D.8). (These local conditions should be supplemented, in the case when the spacetime topology is $R^{4}$, by the non trivial requirement that the field components match at points within a finite distance when coming from infinity in different directions.)

Finally, we note that the transverseness and tracelessness of $p_{a b}$ imply that the surface integral

$$
Q[\xi]=\int \xi^{a} p_{a}{ }^{b} d^{2} \Sigma_{b},
$$


taken over a spacelike two-dimensional section of the "cylinder" $R \times S^{2}$, is actually independent of the chosen section whenever $\xi^{a}$ is one of the ten conformal Killing vectors of the metric (D.8). This is no accident because one can show that the $\mathrm{O}(3,2)$ charges of Sect. III are intimately connected with those defined by (D.24). [The connection involves conjugation ("similarity transformation") of the $\mathrm{O}(3,2)$ charges by an appropriate section-dependent element of the anti-de Sitter group.] Incidentally, one may show that $p_{a b}$ is equal to the electric part of the rescaled Weyl tensor of (D.1). If this fact is used, the charges (D.24) coincide with those considered in [32].

It is important to realize that the conservation of the charges $Q[\xi]$ does not completely follow from the asymptotic behavior (II.2) of the spacetime metric. Indeed, as we have just seen, $Q[\xi]$ will be conserved only if the "constraint equations" (D.15) and (D.16) - which are part of Einstein equations for the physical metric - hold. But (D.15) and (D.16) yield the additional restrictions (D.22) and (D.23) on the leading order of the deviation $h_{\lambda \mu}(t, r, \theta, \phi)$ from the antide Sitter background.

The need for additional restrictions on $h_{\lambda \mu}$ besides those of Sect. II also arises in the hamiltonian formalism. Indeed, as shown in the next appendix, the canonical generators $H[\xi]$ associated with asymptotic $\mathrm{O}(3,2)$ Killing vectors preserve the boundary conditions on the canonical variables, and hence, are well defined as canonical generators, only if additional requirements are met at infinity. From the hamiltonian viewpoint, this happens even on-shell, because there exist solutions of the vacuum Einstein equations which are asymptotic to anti-de Sitter space [in the sense of (II.2e-j), (II.9)] on some spacelike slice, but which do not remain asymptotically anti-de Sitter in the course of evolution.

It should be noted that the necessity of extra boundary conditions in the $(3+1)$-analysis of the field equations in the presence of reflective boundary conditions is not peculiar to the gravitational field. It also occurs, for example, in the simpler case of a two-dimensional Klein-Gordon field $\phi$ propagating in a finite interval $[a, b]$ and required to vanish at the boundaries. In that instance, one finds that the conditions $\phi(a)=\phi(b)=0$ are preserved by the Klein-Gordon equations only if all the spatial derivatives $\phi^{n /}$ of even order also vanish at the endpoints.

\section{Appendix E. Asymptotic Conditions and Time Evolution}

In the main text, we have shown that a spacetime metric obeying the boundary conditions (II.1-2) is asymptotically invariant under spacetime changes of coordinates (or "diffeomorphisms") which become asymptotically anti-de Sitter transformations. We have also shown that in such a spacetime, the spatial metric and its canonical momentum fall off as in (II.2e-2j,9) on the appropriate spacelike sections.

One can then ask the following initial value problem: suppose one is given on an initial surface $t=0$ data $\left(g_{i j}, \pi^{i j}\right)$ which obey the constraint equations everywhere and which have the asymptotic behavior of Sect. II. Can one find appropriate lapse and shift functions such that these initial data can be developed, by means of Hamiltonian equations, into a spacetime metric obeying (II.2)? 
Here, we give to the terms lapse and shift their standard meaning

$$
\begin{aligned}
N^{\perp} & =\left(-g^{00}\right)^{-1 / 2}, \\
N^{i} & ={ }^{(3)} g^{i j} g_{j_{0}} .
\end{aligned}
$$

They are equal to the $\xi^{\perp}$ and $\xi^{i}$ used in the main text when the asymptotic Killing vector is chosen to be $U_{51}$. For simplicity, we restrict the discussion in this appendix to ordinary (vacuum) gravity. The extension to supergravity should proceed along similar lines but we have not investigated it.

The analysis of this problem is much subtler and difficult than its analog in asymptotically flat space. The difficulties which appear may already be seen if one takes as initial data those of a $t=$ constant slice of the Schwarzschild anti-de Sitter metric [Eq. (B.1) with $a=0$ ]. In that case the exact $N^{\perp}, N^{k}$ are of course such that the boundary conditions are preserved in time. However if one takes instead those of anti-de Sitter space, which we will call $N_{a d S}, N_{a d S}^{k}$, one finds that the boundary conditions are not preserved.

Now, the difference between both choices of $N^{\perp}, N^{k}$ vanishes at large distances but, as the above observation shows, cannot be neglected. This means that in the general case one must also impose conditions on the next orders in an expansion of $N^{\perp}$ and $N^{k}$ in decreasing powers of $r$. This, of course, must be done by requiring the permissible $N^{\perp}$ and $N^{k}$ to preserve the boundary conditions (II.2.9) on the canonical variables.

The analysis proceeds as follows. Comparison of Eqs. (II.2) and (E.1) yields the following asymptotic behavior for the lapse and the shift:

$$
\begin{gathered}
N^{\perp}=N_{a d S}^{\perp}+O\left(r^{-2}\right), \\
N^{r}=N_{a d S}^{r}+O\left(r^{-2}\right), \\
N^{\theta}=N_{a d S}^{\theta}+O\left(r^{-3}\right), \\
N^{\phi}=N_{a d S}^{\phi}+O\left(r^{-3}\right) .
\end{gathered}
$$

Straightforward examination of the hamiltonian equations reveal that the $r^{-3}$ and $r^{-4}$ orders of the differences $N^{\theta}-N_{a d S}^{\theta}$ and $N^{\phi}-N_{a d S}^{\phi}$ are completely determined by demanding that $\dot{g}_{r \theta}$ and $\dot{g}_{r \phi}$ (equal to $\left[g_{r \theta}, H[\xi]\right]$ and $\left[g_{r \phi}, H[\xi]\right]$, respectively) be of the same order as $g_{r \theta}$ and $g_{r \phi}$ [i.e., $\left.O\left(r^{-4}\right)\right]$. The other $\dot{g}_{a b}$ equations give no constraint on $N^{\perp}, N^{k}$.

It remains to impose the conditions that $\dot{\pi}^{a b}$ be also of the appropriate order. This should yield restrictions on $N^{\perp}-N_{a d s}^{\perp}$ and $N^{r}-N_{a d s}^{r}$. A problem arises here, however, namely that the conditions $\left[\pi^{a b}, H[\xi]\right]=O\left(\pi^{a b}\right)$ are not two in number, but rather they are four asymptotic partial differential equations. [Four and not six because two of them, the $\dot{\pi}^{r \theta}=O\left(r^{-2}\right)$ and $\dot{\pi}^{r \theta}=O\left(r^{-2}\right)$ equations, are automatically satisfied.] Moreover, $N^{r}$ drops out from the remaining four relations so that they become simultaneous restrictions on the single function $N^{\perp}$.

Now, these equations are not integrable unless the initial data $g_{a b}$ and $\pi^{a b}$ obey additional requirements at infinity. Only if these integrability conditions are met will $N^{\perp}$ exist.

This is not the end of the story for one must impose the preservation in time (i.e., by the hamiltonian equations) of the integrability conditions. This leads to new asymptotic conditions on the canonical variables. 
We will not analyze in detail here the additional asymptotic conditions that must be imposed on the canonical variables. One may be sure that a consistent set of additional restrictions can be found just from the simple observation that those - yet to be determined - conditions will be obeyed by canonical variables $\left(g_{a b}, \pi^{a b}\right)$ that can be obtained from a spacetime metric $g_{\lambda \mu}$ (i) which obeys the boundary conditions given in Sect. II and (ii) which is a solution to Einstein equations.

Moreover the convergence and proporties of the surface integral giving the $\mathrm{O}(3,2)$ charges has been shown without appealing to the explicit form of these extra conditions - although it was implicitly used that the generators $H[\xi]$ mapped allowed configurations on allowed configurations - so the analysis and conclusions in the paper remain valid.

Lastly we remark that the considerations of this section may be carried through if one assumes that the constraint equations are only valid asymptotically (instead of everywhere). One then finds, in particular, that the constraint functions $\mathscr{K}_{\perp}$ and $\mathscr{K}_{k}$ must decrease faster than what would be naively suggested by (II.2). Actually it turns out that they must decrease more rapidly than any power of $r^{-1}$. [This, incidentally, guarantees that the volume integral $\int\left(N^{\perp} \mathscr{K}_{\perp}+N^{k} \mathscr{K}_{k}\right) d^{3} x$ in the Hamiltonian converges - a property which does not follow from (II.2) alone.]

A complete analysis of this problem in $2+1$ spacetime dimensions may be found in [37]. In that simpler theory one finds the concise statement that all the extra conditions are just the requirements on the asymptotic decrease of $\mathscr{K}_{\perp}$ and $\mathscr{K}_{k}$ mentioned above.

Acknowledgements. One of us (C.T.) would like to thank Patricio Cordero for many enlightening and informative discussions on the subject of this article which took place in Princeton in 1977. We are also grateful to A. Ashtekar, P. Candelas, B. Carter, M. Demianski, P. Townsend, and $\mathrm{S}$. Weinberg for useful discussions and correspondence. This work has been supported in part by NSF Grant No. PHY-8216715 to The University of Texas at Austin, by research funds of The University of Texas Center for Theoretical Physics, and by a grant of the Tinker Foundation to the Centro de Estudios Cientificos de Santiago.

Note added. After completion of this work, we were informed that asymptotically anti-de Sitter spaces have been studied along different lines in [32].

\section{References}

1. Regge, T., Teitelboim, C.: Role of surface integrals in the Hamiltonian formulation of general relativity. Ann. Phys. (N.Y.) 88, 286 (1974)

2. Hawking, S.W., Ellis, G.F.R.: The large scale structure of spacetime. Cambridge, UK: Cambridge University Press 1973

3. Carter, B.: Hamilton-Jacobi and Schrödinger separable solutions of Einstein's equations. Commun. Math. Phys. 10, 280 (1968); Demianski, M.: Acta Astron. 23, 211 (1973)

4. Hawking, S.W.: The boundary conditions for gauged supergravity. Phys. Lett. 126 B, 175 (1983)

5. Abbott, L.F., Deser, S.: Stability of gravity with a cosmological constant. Nucl. Phys. B 195, 76 (1982)

6. Breitenlohner, P., Freedman, D.Z.: Stability in gauged extended supergravity. Ann. Phys. (N.Y.) 144, 249 (1982)

7. Gibbons, G.W., Hull, C.M., Warner, N.P.: The stability of gauged supergravity. Nucl. Phys. B 218, 173 (1983)

8. Teitelboim, C.: Surface integrals as symmetry generators in supergravity theory. Phys. Lett. 69 B, 240 (1977); Deser, S., Teitelboim, C.: Supergravity has positive energy. Phys. Rev. Lett. 39, 249 (1977)

9. Witten, E.: A new proof of the positive energy theorem. Commun. Math. Phys. 80, 397 (1981) 
10. Horowitz, G.T., Strominger, A.: Witten's expression for gravitational energy. Phys. Rev. D 27, $2793(1983)$

11. Deser, S.: Positive classical gravitational energy from classical supergravity. Phys. Rev. D 27, 2805 (1983)

12. Hull, C.M.: The positivity of gravitational energy and global supersymmetry. Commun. Math. Phys. 90, 545 (1983)

13. Teitelboim, C.: Manifestly positive-energy expression in classical gravity: simplified derivation from supergravity. Phys. Rev. D 29, 2763 (1984)

14. Aurilia, A., Nicolai, H., Townsend, P.K.: Hidden constants: The $\theta$ parameter of QCD and the cosmological constant of $N=8$ supergravity. Nucl. Phys. B 176, 509 (1980)

15. Henneaux, M., Teitelboim, C.: Phys. Lett. 142 B, 355 (1984)

16. Henneaux, M., Teitelboim, C.: Phys. Lett. 143 B, 415 (1984)

17. Benguria, R., Cordero, P., Teitelboim, C.: Aspects of the Hamiltonian dynamics of interacting gravitational gauge and Higgs fields with applications to spherical symmetry. Nucl. Phys. B 122, 61 (1977)

18. Ashtekar, A.: In: General relativity and gravitation: one hundred years after the birth of Albert Einstein. Held, A. et al.: New York: Plenum Press 1980

19. Penrose, R.: In: Relativity, groups, and topology. DeWitt, C., DeWitt, B. eds. New York: Gordon and Breach 1964

20. Avis, S.J., Isham, C.J., Storey, D.: Quantum field theory in anti-de Sitter spacetime. Phys. Rev. D 18, 3565 (1978)

21. Dirac, P.A.M.: The theory of gravitation in Hamiltonian form. Proc. Roy. Soc. A246, 333 (1958)

22. Arnowitt, R., Deser, S., Misner, C.W.: In: Gravitation: an introduction to current research. Witten, L. ed. New York: Wiley 1962

23. Hanson, A., Regge, T., Teitelboim, C.: Constrained Hamiltonian systems. Acc. Naz. dei Lincei, Rome, 1976

24. Teitelboim, C.: How commutators of constraints reflect the spacetime structure. Ann. Phys. (N.Y.) 79, 542 (1973). See also Teitelboim, C.: In: General relativity and gravitation: one hundred years after the birth of Albert Einstein, Vol. I. Held, A. ed. New York: Plenum Press 1980

25. Dirac, P.A.M.: Lectures on quantum mechanics. Belfer graduate school of science, Yeshiva University, New York, 1964

26. Deser, S., Zumino, B.: Broken supersymmetry and supergravity. Phys. Rev. Lett. 38, 1433 (1977)

27. Townsend, P.: Cosmological constant in supergravity. Phys. Rev. D 15, 2802 (1977)

28. Candelas, P., Raine, D.J.: From anti-de Sitter to Minkowski space via vacuum fluctuations. University of Teyas preprint (1983)

29. 't Hooft, G.: Magnetic monopoles in unified gauge theories. Nucl. Phys. B 79, 276 (1974) Polyakov, A.M.: Particle spectrum in quantum field theory. JETP Lett. 20, 194 (1974)

30. Jackiw, R.: Acta Phys. Austriaca, Suppl. XXII, 383 (1980)

31. Henneaux, M.: Remarks on spacetime symmetries and non-abelian gauge fields. J. Math. Phys. 23, 830 (1982)

32. Ashtekar, A., Magnon, A.: Asymptotically anti-de Sitter spacetimes. Classical and Quantum Gravity Lett. 1, L 39 (1984)

33. Kosmann, Y.: Ann. Mat. Pur. Appl. XCI, 319 (1972)

34. Henneaux, M.: Gen. Rel. Grav. 12, 137 (1980)

35. Cordero, P., Teitelboim, C.: Remarks on supersymmetric black holes. Phys. Lett. 78B, 80 (1978)

36. Eisenhart, L.P.: Riemannian geometry. Princeton, NJ: Princeton University Press 1964

37. Brown, J.D., Henneaux, M.: In preparation

Communicated by S. W. Hawking

Received June 18, 1984; in revised form September 25, 1984 